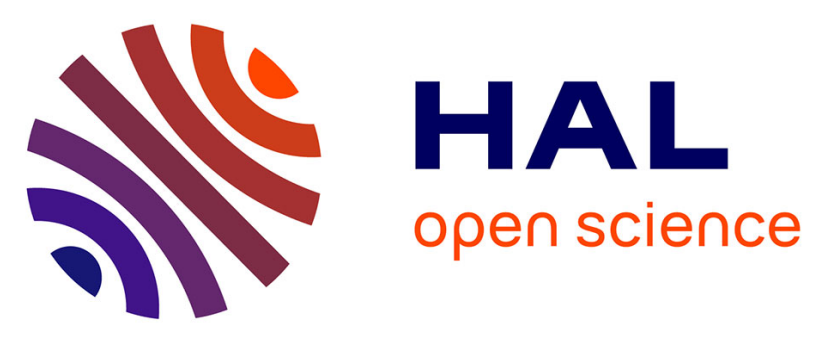

\title{
Sub-pixellic Methods for Sidelobes Suppression and Strong Targets Extraction in Single Look Complex SAR Images
}

Rémy Abergel, Loïc Denis, Saïd Ladjal, Florence Tupin

\section{To cite this version:}

Rémy Abergel, Loïc Denis, Saïd Ladjal, Florence Tupin. Sub-pixellic Methods for Sidelobes Suppression and Strong Targets Extraction in Single Look Complex SAR Images. IEEE Journal of Selected Topics in Applied Earth Observations and Remote Sensing, 2018, 11 (3), 10.1109/JSTARS.2018.2790987 . hal-01570857v2

\section{HAL Id: hal-01570857}

https://hal-imt.archives-ouvertes.fr/hal-01570857v2

Submitted on 13 Nov 2017

HAL is a multi-disciplinary open access archive for the deposit and dissemination of scientific research documents, whether they are published or not. The documents may come from teaching and research institutions in France or abroad, or from public or private research centers.
L'archive ouverte pluridisciplinaire HAL, est destinée au dépôt et à la diffusion de documents scientifiques de niveau recherche, publiés ou non, émanant des établissements d'enseignement et de recherche français ou étrangers, des laboratoires publics ou privés. 


\title{
Sub-pixellic Methods for Sidelobes Suppression and Strong Targets Extraction in Single Look Complex SAR Images
}

\author{
Rémy Abergel, Loïc Denis, Saïd Ladjal and Florence Tupin
}

\begin{abstract}
SAR images display very high dynamic ranges. Man-made structures (like buildings or power towers) produce echoes that are several orders of magnitude stronger than echoes from diffusing areas (vegetated areas) or from smooth surfaces (e.g., roads). The impulse response of the SAR imaging system is thus clearly visible around the strongest targets: sidelobes spread over several pixels, masking the much weaker echoes from the background. To reduce the sidelobes of the impulse response, images are generally spectrally apodized, trading resolution for a reduction of the sidelobes. This apodization procedure (global or shiftvariant) introduces spatial correlations in the speckle-dominated areas which complicates the design of estimation methods. This paper describes strategies to cancel sidelobes around point-like targets while preserving the spatial resolution and the statistics of speckle-dominated areas. An irregular sampling grid is built to compensate the sub-pixel shifts and turn cardinal sines into discrete Diracs. A statistically grounded approach for point-like target extraction is also introduced, thereby providing a decomposition of a single look complex image into two components: a speckle-dominated image and the point-like targets. This decomposition can be exploited to produce images with improved quality (full resolution and suppressed sidelobes) suitable both for visual inspection and further processing (multi-temporal analysis, despeckling, interferometry).
\end{abstract}

Index Terms-SAR imaging, sub-pixel target detection, apodization, sidelobe reduction, speckle, a contrario methodology.

\section{INTRODUCTION}

Synthetic aperture radar (SAR) images offer insight about the back-scattering mechanisms at hand when radar pulses are emitted towards a scene. In particular, two different types of mechanisms generally occur: (i) man-made structures generate multiple bounces that lead to both very high amplitude and well-localized echoes in the SAR images; (ii) in vegetated areas, numerous echoes are produced, these echoes interfere, leading to a speckle phenomenon. Hence, a typical SAR image like the one shown in Fig. 1 (a) contains rather homogeneous areas with fluctuations due to speckle phenomenon (the larger the average intensity in the area, the larger are these fluctuations) and the signature of man-made structures in the form of intensities that are several orders of magnitude larger.

Visualization of such high dynamic range images requires clipping values above some threshold (as done to produce Fig. 1 (a)). Because the strongest echoes are several orders of magnitude more intense than that of the surrounding areas, the impulse response of the complete SAR imaging system (comprised of the SAR sensor and the digital synthesis of the SAR image) is visible. A trade-off must then be found between resolution preservation (limited widening of the impulse response) and attenuation of the sidelobes of the impulse response.

Images delivered by spatial agencies underwent a Fourier apodization process to prevent the strongest echoes to spread over several tens of pixels in a cross shape (the typical SAR impulse response without apodization). Beyond a resolution loss, noticeable by the reduced ability to separate two close-by echoes, this apodization impacts regions dominated by speckle. While, without neither apodization

Rémy Abergel, Saïd Ladjal and Florence Tupin are with LTCI, Télécom ParisTech, 75013 Paris, France (e-mails: firstname.lastname@telecomparistech.fr). Loïc Denis is with the Univ Lyon, UJM-Saint-Etienne, Institut d'Optique Graduate School, Laboratoire Hubert Curien CNRS UMR 5516, F-42023 Saint-Etienne, France (e-mail: loic.denis@univ-st-etienne.fr). nor oversampling, fluctuations within speckle-dominated regions are statistically independent, apodization (and over-sampling) introduces spatial correlations in these fluctuations. Visually, these fluctuations appear grainy, but, more importantly, these correlations impact statistical methods for speckle reduction. Almost all these methods assume that speckle is not spatially correlated [1]. Even methods that are relatively immune to speckle correlation (e.g., NL-SAR [2]) behave better in the absence of these correlations. Two options are generally considered to handle speckle correlations: (i) subsampling the apodized image to reduce the spatial correlations, and (ii) speckle whitening, by processing the unapodized (and, if necessary, resampled at Nyquist rate) image [3], similarly as done in image processing to denoise images suffering from correlated noise [4]. None of these two approaches is very satisfactory: the first approach involves a resolution loss while the second, by removing the apodization, reinforces the impulse response sidelobes so that the resulting image is corrupted by large cross-shaped streaks around brightest pixels.

Sidelobes reduction in SAR imagery has been the subject of several works. Beyond apodization (i.e., linear processing by spectral weighting), two main approaches have been proposed to improve images with very strong echoes such as those created by ships or buildings: nonlinear spatially variant apodization methods and target extraction techniques.

The aim of spatially variant apodization methods is to reduce sidelobes without sacrificing the spatial resolution, i.e., without widening the main lobe. Nonlinear processing methods were introduced in [5] under the name Spatially Variant Apodization (SVA) to reach this goal. The starting point is the observation that, by non-linearly combining impulse responses from a family of spectral weighting functions (e.g., cosine-on-pedestal weighting functions that include Hann and Hamming apodizations as special cases) an improved impulse response can be obtained. All impulse responses are maximum at the location of the target, they then differ in terms of sidelobes amplitude (reduced when the apodization is stronger) and main lobe width (narrowest in the absence of apodization). By retaining at each pixel the smallest amplitude among all amplitudes obtained by applying the family of apodization functions, sidelobes are kept minimum while preventing the widening of the main lobe. The choice of cosine-onpedestal apodization functions leads to a very efficient algorithm that can be applied in the signal domain as a space-variant finite impulse response filter with very compact response [5]. Several extensions of SVA were later introduced, in particular to perform super-resolution, see e.g. [6]. We show in this paper that, despite its attractive computational efficiency, SVA suffers from several drawbacks: (i) it modifies the statistics of speckle-dominated areas, (ii) point-like targets are still spread over several pixels, (iii) a negative bias is introduced (homogeneous regions appear to have a lower reflectivity after SVA processing). A somewhat related method for sidelobes reduction, also based on a filter bank, has been investigated in [7]. Rather than locally selecting the best suited apodization, spectral super-resolution techniques (Capon [8] or APES [9]) are applied to improve the localization of point targets. These spectral superresolution techniques require an estimation of the signal covariance. 
After decomposition of the original SLC image into small patches (typically $32 \times 32$ ), many sub-aperture images are generated for each patch and the covariance is estimated based on these sub-aperture images. A super-resolved patch is then produced and the final superresolved image is obtained by mosaicking all processed patches. This method is computationally expansive but produces super-resolved images with strongly reduced sidelobes provided that the signal covariance can be correctly estimated and inverted. However, bright targets are not explicitly detected nor extracted, speckle-corrupted areas appear to be affected by the process (the super-resolution introduces strong spatial correlations), and extended targets tend to be thinned by the super-resolution process. This method is probably not applicable as a general-purpose sidelobe reduction step prior to subsequent automated processing.

The second approach to sidelobe reduction is based on the detection of the strongest targets in order to extract them from the SAR image. The residual image then contains speckle-dominated regions that can be further processed, for example by a speckle reduction method. This is the approach followed by [10] and some other denoising methods, see the discussion in section V.C of [1].

Target detection in SAR images is an extensively studied topic. In particular, many works are devoted to identifying weak targets or moving targets [11]-[15], under foliage targets [16], using polarimetric data [17]-[19] or interferometric data [20], [21]. In the context of image contamination by the sidelobes of strong targets, the difficulty does not lie in the detection of the target (since the signal-to-noise ratio is very high), but on the necessity to perform subpixel localization of the target to correctly account for the sidelobes. Detection techniques based on CLEAN algorithm [22]-[24] and its extension RELAX [25] are then well-adapted. They process a SAR image iteratively, detecting an additional target at each iteration from the residuals obtained by subtracting already detected target signatures from the SAR image. Each time a target is found, the amplitude and sub-pixel location of the target are estimated in the maximum likelihood sense, i.e., such that the residuals are minimized.

Decomposition models of a SAR image into strong scatterers and homogeneous regions have been proposed [26], [27]. These decomposition approaches however do not take into account the sidelobes of strong scatterers (they apply to images that are apodized).

Contributions: this paper extends the recent conference paper [28] and introduces:

(i) an irregular resampling procedure that suppresses sidelobes of strong targets; this procedure preserves the statistical properties of speckled areas and prevents from spreading bright targets,

(ii) a criterion based on the statistical framework of a contrario methods to detect targets in speckle, with an explicit control over the number of false alarms in the image,

(iii) a decomposition method that can separate a SAR image into two components: one with speckle-dominated areas, the other with all the strong targets; these components can be further processed in order to reduce speckle or to improve the resolution.

The paper is organized as follows. Section II describes the spectral apodization and oversampling that are typical in single look complex (SLC) SAR images. Section III introduces a criterion to identify the sub-pixel translation that would suppress at best the sidelobes. This criterion is then applied to obtain an irregular resampling scheme that produces images with strongly reduced sidelobes. Section IV derives a target detection method. This method leads to a decomposition scheme into a speckle-dominated component and a target component whose efficiency and practical interest is illustrated in several experiments.

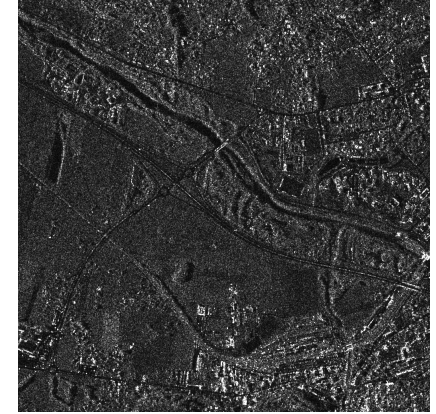

(a) amplitude image $|u|$

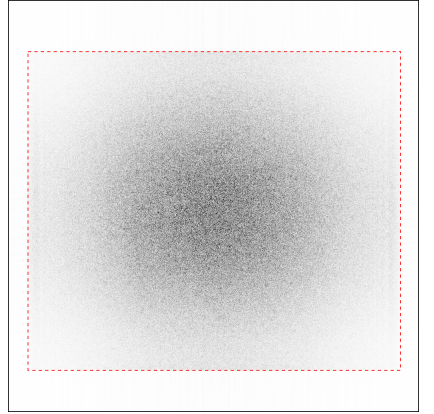

(b) Fourier modulus $|\widehat{u}|$
Fig. 1. An example of TerraSAR-X image data. We display in (a) the modulus of a SLC TerraSAR-X image $u$ with size $M \times N$ (by convention in all this work, the horizontal axis represents the range direction), and in (b) the modulus of its DFT $\widehat{u}$ (low values are displayed in bright and high values are displayed in dark). The Fourier spectrum (b) vanishes outside a rectangular sub-frequency domain $\widehat{\omega}$ with size $m \times n$ (delimited by the red dashed line), showing that the image $u$ is oversampled by a factor $M / m$ in the range direction, and by a factor $N / n$ in the azimuth direction.

\section{PSEUDO-RAW IMAGE AND PSEUDO-RAW SPECTRUM}

Let $u: \Omega \rightarrow \mathbb{C}$ be a SLC discrete image with size $M \times N$ and spatial domain $\Omega=I_{M} \times I_{N}$, noting $I_{K}=\{0, \ldots, K-1\}$. We denote by $\widehat{u}$ the discrete Fourier transform (DFT) of $u$, which is the two-dimensional and $(M, N)$-periodic complex-valued signal defined by

$$
\forall(\alpha, \beta) \in \mathbb{Z}^{2}, \quad \widehat{u}(\alpha, \beta)=\sum_{(k, \ell) \in \Omega} u(k, \ell) e^{-2 i \pi\left(\frac{k \alpha}{M}+\frac{\ell \beta}{N}\right)} .
$$

Generally, the study of $\widehat{u}$ is restricted to the period $\widehat{\Omega}=\widehat{I}_{M} \times \widehat{I}_{N}$, noting $\widehat{I}_{K}=\left[-\frac{K}{2}, \frac{K}{2}\right) \cap \mathbb{Z}$. The domain $\widehat{\Omega}$ is called the canonical frequency domain (or reciprocal grid) associated to $\Omega$.

The SLC SAR images are, for reasons due to their acquisition process, band-limited and well-sampled signals. This is illustrated in Fig. 1, in which we display the modulus of a SLC TerraSAR-X image $u$ (Fig. 1 (a)) and the modulus of its DFT $\widehat{u}$ (Fig. 1 (b)). Indeed, the restriction of $\widehat{u}$ to $\widehat{\Omega}$ is zero-valued everywhere outside a rectangular frequency domain $\widehat{\omega}:=\widehat{I}_{m} \times \widehat{I}_{n} \subset \widehat{\Omega}$ of size $m \times n$, showing that the pixel spacing was adjusted during the sampling process to (over) satisfy the Shannon-Nyquist criterion. Besides, and as described in [29], the non-zero part of the Fourier spectrum is in fact apodized, which means that for any $(\alpha, \beta) \in \widehat{\Omega}$, we have

$$
\widehat{u}(\alpha, \beta)=\left\{\begin{array}{cl}
\widehat{u_{0}}(\alpha, \beta) \cdot \gamma(\alpha, \beta) & \text { if }(\alpha, \beta) \in \widehat{\omega} \\
0 & \text { otherwise, }
\end{array}\right.
$$

where $\gamma: \widehat{\omega} \rightarrow \mathbb{R}_{+} \backslash\{0\}$ is a frequency attenuating function which depends on the data provider, and we name $\widehat{u_{0}}: \widehat{\omega} \rightarrow \mathbb{C}$ the pseudoraw spectrum. The complex-valued image $u_{0}: \omega \rightarrow \mathbb{C}$ with spatial domain $\omega:=I_{m} \times I_{n}$ is hereafter called the pseudo-raw image. Since the images $u$ and $u_{0}$ have different pixel sizes, the pseudo-raw image $u_{0}$ will always be compared to the subsampled image $u_{\omega}: \omega \rightarrow \mathbb{C}$ obtained by resampling $u$ at the Nyquist frequency, i.e. the image defined in the Fourier domain by

$$
\forall(\alpha, \beta) \in \widehat{\omega}, \quad \widehat{u_{\omega}}(\alpha, \beta)=\frac{|\omega|}{|\Omega|} \cdot \widehat{u}(\alpha, \beta),
$$

where $|\omega|=m \cdot n$ and $|\Omega|=M \cdot N$ denote the cardinality of the sets $\omega$ and $\Omega$.

In the particular case of TerraSAR-X [29], the apodization $\gamma$ is, up to a multiplicative factor $a_{\gamma} \in \mathbb{R}$ that we introduce to ensure 


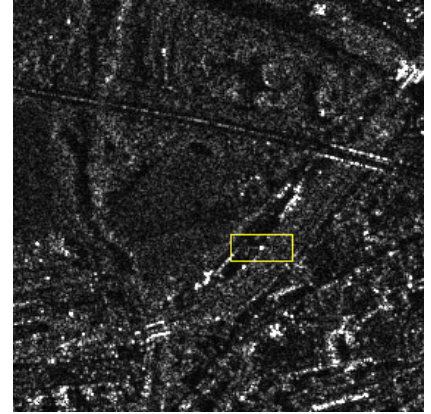

(a) apodized image $u_{\omega}$

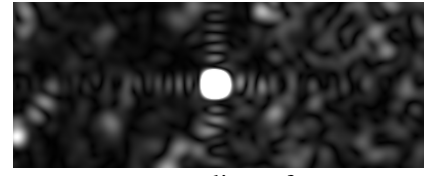

oversampling of $u_{\omega}$

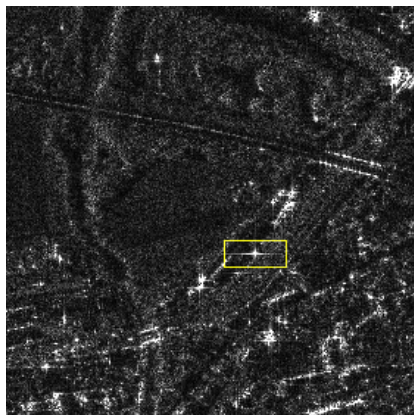

(b) pseudo-raw image $u_{0}$

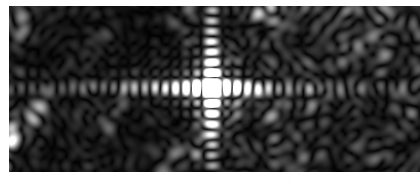

oversampling of $u_{0}$
Fig. 2. Comparison between apodized and pseudo-raw images. On the first row, we display the modulus of a portion of the apodized image $u_{\omega}$ and the pseudo-raw image $u_{0}$. Without apodization (pseudo-raw image $u_{0}$ ) the spatial resolution is higher but strong targets lead to sidelobes that mask out the surrounding structures. The magnifications (zooming with factor ten by Shannon interpolation) shown on the second row illustrate this trade-off between widening of the central lobe and attenuation of the sidelobes (the images are displayed using a common grayscale).

that both amplitude images $\left|u_{0}\right|$ and $\left|u_{\omega}\right|$ have the same maximum, a 2D-separable Hamming function,

$$
\begin{aligned}
\forall(\alpha, \beta) \in \widehat{\omega}, \quad \gamma(\alpha, \beta)=a_{\gamma} \cdot \gamma_{m}(\alpha) \cdot \gamma_{n}(\beta), \quad \text { where } \\
\gamma_{K}(\xi)=\lambda+(1-\lambda) \cos \left(\frac{2 \pi \xi}{K}\right), \quad \text { and } \quad \lambda=0.6,
\end{aligned}
$$

so that the unapodized pseudo-raw spectrum $\widehat{u_{0}}$ can be easily recovered from the initial spectrum $\widehat{u}$ by inverting (2). We refer the reader to [3], [28] for more details about the computation of the pseudo-raw image $u_{0}$ from $u$ (in particular in the case when the frequency attenuating function $\gamma$ is unknown).

The reason why the spatial agencies introduce an apodization is to attenuate the sidelobes of the strong point target responses (highly present in urban areas) which are visible due to the SAR impulse response. Indeed, an unresolved and isotropic target with constant reflectivity over the radar electromagnetic band can be modeled after the synthesis of the SAR image by a $2 \mathrm{D}$ separable cardinal sine whose sidelobes remain particularly visible in the vicinity of the target center, as we observe in Fig. 2 (b). Unfortunately, the reduction of those sidelobes using apodization also results in a degradation of the spatial resolution (see Fig. 2 (a)) and the choice of using the Hamming apodization (4) in the case of TerraSAR-X is presented in [29] as a trade-off between sidelobe reduction and deterioration of the resolution.

\section{AN IRREGULAR RESAMPLING SCHEME FOR COMPLEX PSEUDO-RAW SAR IMAGES}

In this section, we complete with more details and experimental results our previous work presented in [28], and we discuss the strengths and weaknesses of the proposed irregular resampling scheme.

\section{A. The cardinal sine impulse response model}

In stripmap mode, the SAR imaging system (acquisition + SAR synthesis) exhibits an approximate separability in range and azimuth, with rectangular spectra in both dimensions. Consequently, the impulse response of the system is very well approached by a twodimensional product of cardinal sine functions,

$$
\forall(x, y) \in \mathbb{R}^{2}, \quad \operatorname{sinc}(x, y)=\frac{\sin (\pi x)}{\pi x} \cdot \frac{\sin (\pi y)}{\pi y},
$$

with the continuity preserving condition $\frac{\sin (0)}{0}=1$. Notice that in (5), the coordinates $(x, y)$ are scaled in order to set the range and azimuth bandwidths both equal to $2 \pi$ (i.e., the horizontal and vertical dimensions of the spectrum support represented by the red-dashed rectangle in Fig. 1 (b)). Under this model, the continuous signal $U_{0}^{c}: \mathbb{R}^{2} \rightarrow \mathbb{C}$, before sampling, can be modeled as the convolution between the continuous latent scene and the impulse response. It follows that $U_{0}^{c}$ is a band-limited signal which can be reconstructed exactly (neglecting sensor noise), according to the Shannon-Whittaker Sampling Theorem [30], [31], provided an infinite number of its samples $\left\{U_{0}^{c}\left(k \cdot \delta_{\mathrm{r}}, \ell \cdot \delta_{\mathrm{az}}\right)\right\}_{(k, \ell) \in \mathbb{Z}^{2}}$ are observed at regularly spaced locations, with steps $\delta_{\mathrm{r}} \leq 1$ along the range direction and $\delta_{\mathrm{az}} \leq 1$ along the azimuth direction. The pseudo-raw image $u_{0}$ corresponds to the finite sampling of $U_{0}^{c}$ at the critical sampling step, $\delta_{\mathrm{r}}=\delta_{\mathrm{az}}=1$, while the initial image $u$ corresponds to the sampling of $U_{0}^{c}$ using the sub-critical steps $\delta_{\mathrm{r}}=m / M<1$ and $\delta_{\mathrm{az}}=n / N<1$. In the following, we will denote by $U_{0}: \mathbb{R}^{2} \rightarrow \mathbb{C}$ the Shannon interpolate of $u_{0}$ ( $U_{0}$ is different from $U_{0}^{c}$ since $u_{0}$ is made of only a finite number of samples), which can be computed as $U_{0}=U_{0}^{r}+i \cdot U_{0}^{i}$, noting $U_{0}^{r}$ and $U_{0}^{i}$ the (real-valued) Shannon interpolates of the real and imaginary parts of $u_{0}$ (see for instance Definition 2 in [32] for the explicit interpolation formula).

In the rest of this section, we drop the double indexes for the spatial coordinates in order to simplify the equations. The contribution to the pseudo-raw image of an unresolved target (that is, a target with a spatial extension that is negligible with respect to the SAR resolution) located at sub-pixel location $k_{0}+\delta$ is:

$$
\forall k \in \omega, \quad u_{0}(k)=A \operatorname{sinc}\left(k-\left(k_{0}+\delta\right)\right)+u_{0}^{*}(k),
$$

where $A \in \mathbb{C}$ denotes target's amplitude, $k_{0} \in \omega, \delta \in\left[-\frac{1}{2}, \frac{1}{2}\right)^{2}$, and $u_{0}^{*}$ is the signal in the absence of the target. When $\delta \neq 0$, the position of the target center does not coincide with the sampling grid and for large values of $|A|$, the signal $u_{0}^{*}$ is dominated by the oscillations of the cardinal sine function in the vicinity of $k_{0}$.

A straightforward solution to this problem consists in resampling the image $u_{0}$ over a translated grid in which the coordinates of the target center are integers, or equivalently, to translate the image $u_{0}$ by the sub-pixellic translation vector $t=-\delta$. Indeed, the translated image is $v_{0}: k \mapsto U_{0}(k+\delta)$ and satisfies

$$
\forall k \in \omega, \quad v_{0}(k)=U_{0}^{*}(k+\delta)+\left\{\begin{array}{cl}
A & \text { if } k=k_{0} \\
0 & \text { otherwise },
\end{array}\right.
$$

where $U_{0}^{*}$ denotes the Shannon interpolate of $u_{0}^{*}$. The resampled image $v_{0}$ is not anymore polluted by the cardinal sine oscillations present in $u_{0}$, and the image $v_{0}$ corresponds to a resampling of $u_{0}^{*}$, except at the position $k=k_{0}$ where the target appears. This phenomenon is clearly illustrated on a portion of a TerraSAR-X image in Fig. 3, where the profiles extracted from Fig. 3 (a) and Fig. 3 (b) both correspond to a sampling over two translated grids of the cardinal sine function. The noticeable difference between the two sampling grids is that the one used for the blue-dotted line contains the target center so that all the target energy is concentrated into a single sample and the effects of the sidelobes are suppressed.

Unfortunately, when the image $u_{0}$ contains more than one target, a global translation is in general not sufficient to accommodate all the targets at the same time. For that reason, we introduced in [28] 

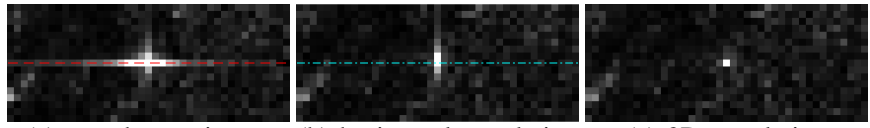

(a) pseudo-raw image

(b) horizontal translation

(c) $2 \mathrm{D}$ translation

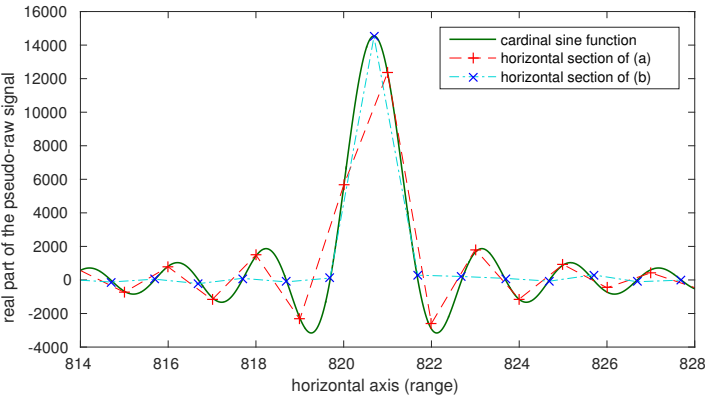

Fig. 3. Profile of a strong target response at the sub-pixellic scale. We display in (a) the modulus of the pseudo-raw image in the vicinity of a strong target and we display in (b) and (c) the modulus of the pseudo-raw image resampled over a translated grid, with translation vector $t=(0.3,0)$ and $t=(0.3,0.1)$ respectively, yielding more localized target support. In the second row, the real part of a range profile of (a) is represented by the red dashed line, while the corresponding profile in (b) is represented by the blue dotted line. The actual sampling of each curve is indicated using some colored plus and cross marks. The green plain curve represents a pure cardinal sine function of type $x \mapsto A^{r} \operatorname{sinc}\left(x-x_{0}\right)$, for some appropriate values of $x_{0} \in \mathbb{R}$ (sub-pixellic range coordinate of the target center) and $A^{r} \in \mathbb{R}$ (real part of the target amplitude). Some similar profiles are observed when we consider the imaginary part of the signal, as well as when we extend the study to the azimuth direction (as can be verified in (c))

an irregular resampling scheme to locally reduce the influence of the sidelobes of the strongest targets.

\section{B. Irregular resampling scheme}

The irregular resampling scheme consists in computing from the pseudo-raw image $u_{0}$ a dense displacement field $T=\left(T_{x}, T_{y}\right)$ : $\omega \rightarrow\left[-\frac{1}{2}, \frac{1}{2}\right] \times\left[-\frac{1}{2}, \frac{1}{2}\right]$, that can be used to irregularly resample $u_{0}$ into the image $v_{0}: \omega \rightarrow \mathbb{C}$ defined by

$$
\forall(k, \ell) \in \omega, \quad v_{0}(k, \ell)=U_{0}\left(k-T_{x}(k, \ell), \ell-T_{y}(k, \ell)\right) .
$$

What we expect from the local displacement $T$ is the following.

(i) In the vicinity of a strong target, the local displacement should cancel the effects of the sidelobes.

(ii) The cancellation of the sidelobes should be as robust as possible to target mixing situations.

(iii) In the regions where no strong target is present, the local displacement field should preserve the speckle statistics, so that posterior processings (such as denoising, segmentation, etc.) remain possible using standard methods with no modification.

The two components $T_{x}$ and $T_{y}$ of the displacement field $T$ will be computed independently, which is motivated by the separability in range and azimuth of the system's impulse response, but also by the drastic reduction of the algorithmic complexity that it offers. We recall below how the computation of the displacement field in range $T_{x}$ is done, that of $T_{y}$ being totally similar (by simply exchanging the two coordinates).

Let $K \in \mathbb{N}$ denote a locality parameter and set $\Delta \omega=[-K, K] \cap \mathbb{Z}$. For any position $\left(k_{0}, \ell_{0}\right) \in \omega$, we will compute $T_{x}\left(k_{0}, \ell_{0}\right)$ using

$$
T_{x}\left(k_{0}, \ell_{0}\right)=\underset{-\frac{1}{2} \leq t \leq \frac{1}{2}}{\operatorname{argmin}} J\left(U_{0}\left(k_{0}-t+\Delta \omega, \ell_{0}\right)\right),
$$

where $J$ (defined below in (10)) is a cost function which is designed to promote the choice of the best horizontal translation according to (i), (ii) and (iii), and $U_{0}\left(k_{0}-t+\Delta \omega, \ell_{0}\right)$ is the mono-dimensional discrete signal of size $2 K+1$ obtained by restricting the $2 \mathrm{D}$ periodical signal $U_{0}: \mathbb{R}^{2} \rightarrow \mathbb{C}$ to the discrete set $\left(k_{0}-t+\Delta \omega\right) \times\left\{\ell_{0}\right\}$. The cost function $J$ is a variant of the discrete total variation operator, whose role is to promote the choice of the translation yielding the mono-dimensional discrete signal $U_{0}\left(k_{0}-t+\Delta \omega, \ell_{0}\right)$ that is the least oscillatory (as is the case of the blue-dotted signal in Fig. 3, which is much less oscillatory than the red-dashed one). It is defined by

$$
\begin{aligned}
& \forall v: \Delta \omega \rightarrow \mathbb{C}, \quad J(v)=\mathrm{TV}_{\text {mask }}^{\mathrm{d}}\left(v^{r}\right)+\mathrm{TV}_{\text {mask }}^{\mathrm{d}}\left(v^{i}\right), \\
& \text { where } \operatorname{TV}_{\text {mask }}^{\mathrm{d}}(s)=\sum_{\substack{-K \leq p<K \\
p \notin\left\{p_{0}(s)-1, p_{0}(s)\right\}}}|s(p+1)-s(p)|,
\end{aligned}
$$

noting $v^{r}$ and $v^{i}$ the real and imaginary parts of $v$, and $p_{0}(s)$ the position where $|s|$ is maximal. The resulting irregular resampling algorithm is given in the box titled Algorithm 1.

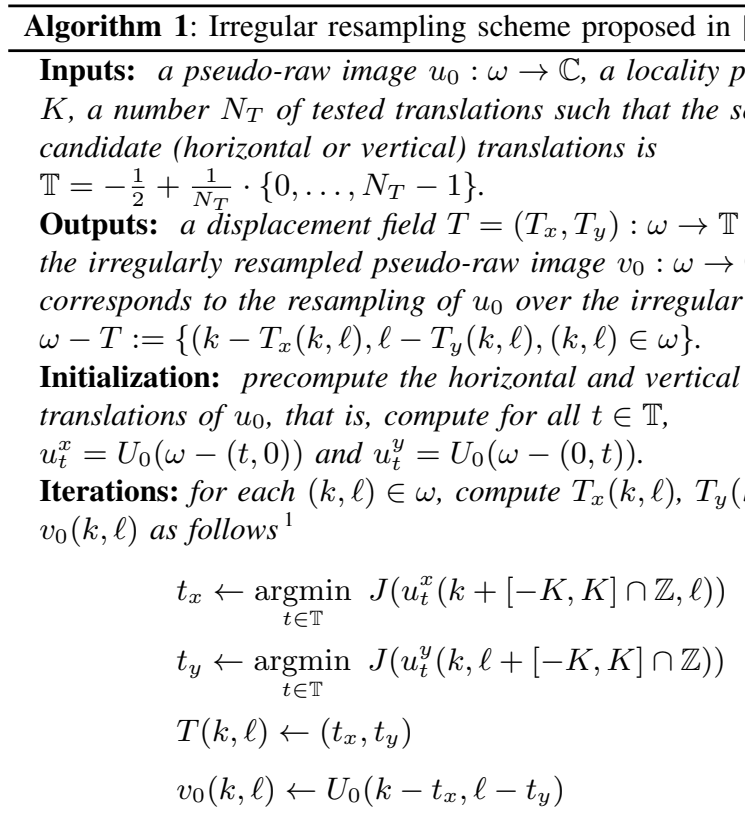

${ }^{1}$ In (11a) and (11b), we use a periodic convention for the images $u_{t}^{x}$ and $u_{t}^{y}$ when their evaluation outside of $\omega$ is needed. In (11d), the value $U_{0}\left(k-t_{x}, \ell-\right.$ $\left.t_{y}\right)$ can be efficiently computed by evaluating the Shannon interpolation of the mono-dimensional signal $\ell \mapsto u_{t_{x}}^{x}(k, \ell)$ at the subpixellic position $\ell-t_{y}$ (this operation simply involves the inner product between the mono-dimensional discrete signal and a cardinal sine function).

First, we illustrate the performance of this resampling method in Fig. 4, where Algorithm 1 is used to resample two pseudo-raw images: a satellite image (TerraSAR-X in stripmap mode, copyright DLR, LAN-1706 project acquired on the region Auvergne-RhôneAlpes, South of France) and an airborne image (RAMSES sensor, ONERA). We show on this experiment that the spatial resolution is preserved by the resampling procedure while suppressing sidelobes that were visible in the unapodized image.

When the complex amplitude in a given resolution cell results from the interferences from several echoes of comparable amplitude, strong intensity fluctuations are observed in the SAR image (the so-called speckle phenomenon [33]). If several acquisitions of the same scene are available, the speckle can be reduced by temporal averaging (a.k.a. multi-looking). More precisely, if $\left\{a^{k}\right\}_{1 \leq k \leq N_{\text {looks }}}$ is a sequence of $N_{\text {looks }}$ complex-valued images, the associated multi-look image is the real-valued image $a^{\mathrm{ML}}=\left(1 / N_{\text {looks }} \cdot \sum_{k=1}^{N_{\text {looks }}}\left|a^{k}\right|^{2}\right)^{0.5}$. In Fig. 5, we compare the multi-look image computed from an apodized sequence 


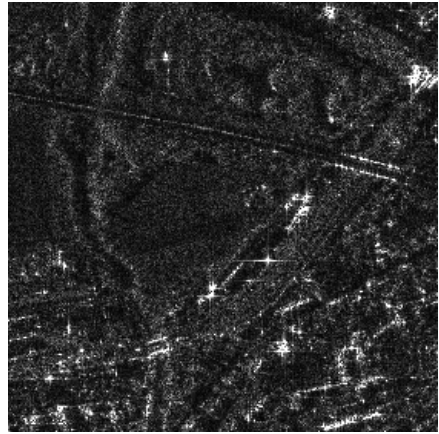

(a) TerraSAR-X pseudo-raw image

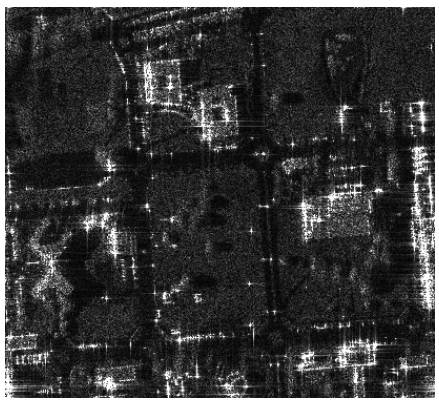

(b) Airborne SAR pseudo-raw image copyright CNES and ONERA

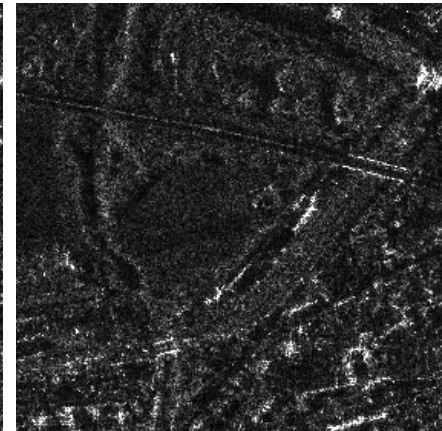

irregular resampling of (a)

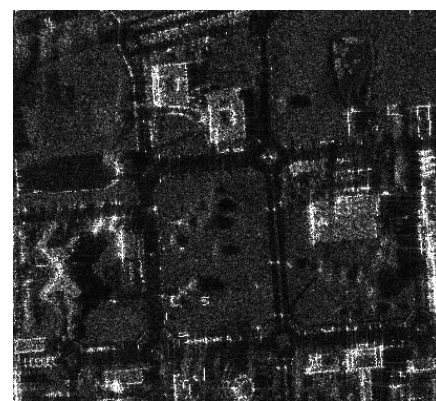

irregular resampling of $(b)$

Fig. 4. Irregularly resampled pseudo-raw images. Images (a) and (b) represent the modulus of two pseudo-raw images, coming from different remote sensing systems (respectively TerraSAR-X and RAMSES airborne SAR system). Both images were irregularly resampled using Algorithm 1, the modulus of the resulting images is displayed in the second column. We observe from this experiment that irregular resampling preserves the spatial resolution while suppressing the sidelobes surrounding bright targets.

of twenty-six TerraSAR-X images (Fig. 5 (a)) to that computed from the corresponding sequences of pseudo-raw images (Fig. 5 (b)), SVA-processed images (Fig. 5 (c)) and irregularly resampled images (Fig. 5 (d)). We can see that Fig. 5 (a) suffers from a significant resolution loss that is mainly visible as a spreading effect of strong targets. The improved resolution of the pseudo-raw sequence is visible in Fig. 5 (b) but strong sidelobes are visible around bright targets, masking the structures located close by. In Fig. 5 (c), we observe an interesting sidelobes removal but the spreading effect of strong targets is still present and this image also suffers from a negative bias of the gray levels (the image is darker). The proposed irregular resampling procedure leads to a multi-look image with preserved spatial resolution and much well-localized targets. Since no noticeable blurring of strong targets can be observed after temporal averaging of twenty-six images processed independently, we conclude that the irregular resampling procedure can efficiently recenter pointlike targets by properly canceling their sub-pixel shift from the pixel center. In addition to the image quality improvements provided by the resampling strategy compared to the use of apodization or SVA, this experiment shows that standard SAR image processing methods (such as, for instance, the reduction of speckle by temporal or spatial averaging) can be used without modification to further process the resampled images, thanks to the preservation of the speckle statistics offered by our resampling strategy, as we shall discuss below.

\section{Advantages and drawbacks}

The proposed irregular resampling strategy provides a simple and efficient alternative to the traditional use of apodization or SVA. We list and discuss below the advantages provided by this resampling scheme.

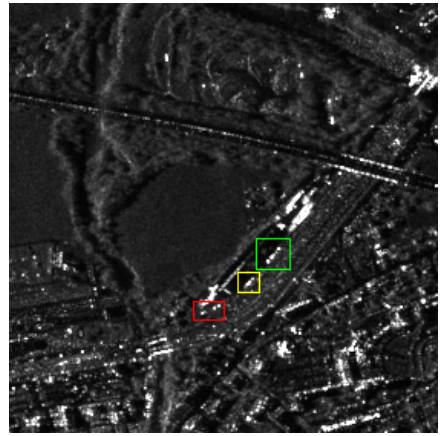

(a) apodized images

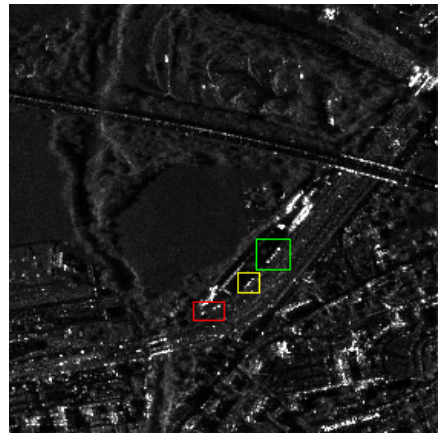

(c) spatially variant apodization

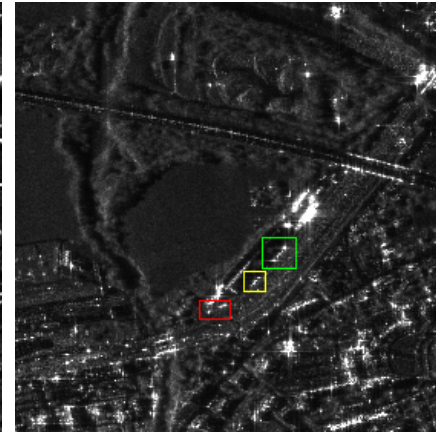

(b) pseudo-raw images

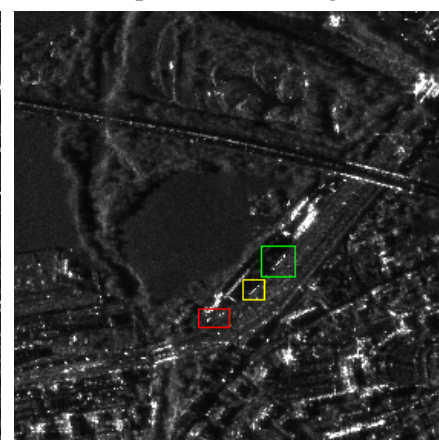

(d) proposed resampling
Fig. 5. Temporal multi-look averages. We display in (a) the multi-look image computed from a sequence $\left\{u_{\omega}^{k}\right\}_{1 \leq k \leq 26}$ of twenty-six TerraSAR$\mathrm{X}$ (apodized) images. By removing the apodization to each image $u_{\omega}^{k}$, we get a sequence of pseudo-raw images $\left\{u_{0}^{k}\right\}_{1 \leq k<26}$ whose corresponding multi-look image is displayed in (b). Computing the multi-look images after applying SVA on each pseudo-raw image $u_{0}^{k}$ yields image (c). By applying Algorithm 1 to each pseudo-raw image $u_{0}^{k}$, we obtain a sequence of irregularly resampled images $\left\{v_{0}^{k}\right\}_{1 \leq k \leq 26}$ and the corresponding multi-look image is displayed in $(\mathrm{d})$.

(i) Preservation of the resolution and sidelobes suppression : the irregular resampling scheme applied to a pseudo-raw image $u_{0}$ boils down to a resampled image $v_{0}$ which is free of the sidelobes induced by the strong target responses while it exhibits a spatial resolution that is similar to that of $u_{0}$. The dense displacement field $T$ used to resample the pseudo-raw image (see (8)) is computed in order to reduce the local oscillations of the signal, according to a discrete total variation-based criterion. Therefore no explicit detection of the targets is involved and the resampling procedure reveals very robust to different target mixing configurations, as illustrated in Fig. 4 and Fig. 5. Notice that the irregular resampling strategy compares favorably to SVA in terms of localization of bright targets and preservation of the dynamic of the pixel values, as illustrated in Fig. 5.

(ii) Preservation of the statistics : one can check that the resampling scheme preserves well the statistics of the pseudo-raw image in areas corresponding to pure and fully developed speckle [34]. Indeed, after the resampling process, the real and imaginary parts of the pixel values of $v_{0}$ remain uncorrelated and follow a Gaussian distribution in areas corresponding to fully developed speckle noise, which is not the case for images processed by SVA, as illustrated in Fig 6. Thanks to this preservation of the statistics offered by the irregular resampling approach, the standard SAR image processing methods can be used without modification to further process the resampled image.

(iii) Simplicity and rapidity : the irregular resampling of a pseudoraw image can be achieved using Algorithm 1 which is simple to implement and has a linear complexity with respect to 


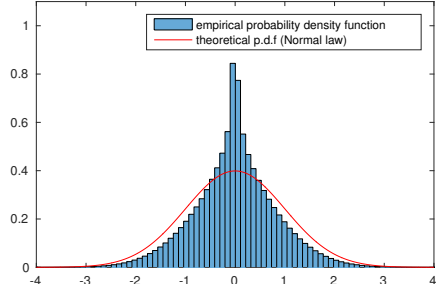

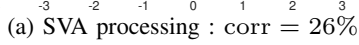

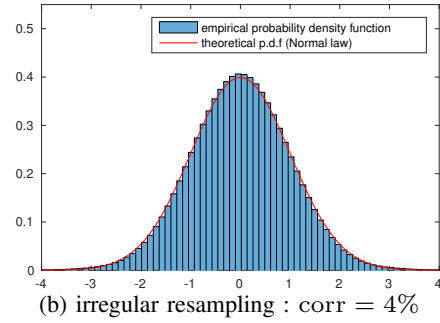

(b) irregular resampling : corr $=4 \%$
Fig. 6. Effect of SVA and irregular resampling on the speckle statistics. In this experiment, we computed a synthetical speckle image $u_{0}$ with size $5000 \times 5000$ and reflectivity 2 , that is, an image whose sequences of real and imaginary parts pixel values $\left\{u_{0}^{r}(x)\right\}$ and $\left\{u_{0}^{i}(x)\right\}$ are made of i.i.d. Gaussian variables with zero mean and unitary variance. We display the empirical probability density function (p.d.f) of the real part of the image obtained after processing $u_{0}$ using SVA (a) or the irregular resampling scheme (b). We also indicate the correlation between neighboring pixels in both processed images using $\operatorname{corr}(w)=\frac{1}{\|w\|^{2}} \sum_{k=0}^{5000-2} \sum_{\ell=0}^{5000-1} w(k+1, \ell) \cdot \bar{w}(k, \ell)$, noting $\bar{w}$ the complex conjugate of $w$.

the image size. More precisely, the complexities in time and memory are $\mathscr{O}\left(N_{T} K|\omega|\right)$ and $\mathscr{O}\left(N_{T}|\omega|\right)$ respectively, after the precomputation of $N_{T}$ FFTs in $\mathscr{O}\left(N_{T} \cdot|\omega| \log |\omega|\right)$ during the initialization step. On a standard computer, a $\mathrm{C}$ implementation of Algorithm 1 processes a pseudo-raw image of size $|\omega|=1000 \times 1000$ in less than two minutes using the typical setting $N_{T}=20$ and $K=25$.

While apodization introduces undesirable correlations of the speckle and deteriorates the spatial resolution, the irregular resampling scheme provides an image with better resolution and uncorrelated speckle, which is suitable both for visualization and further processing. This resampling strategy also presents some limitations that we shall discuss now and that suggest the development of a method based on the extraction of bright targets, as proposed in section IV.

(i) Non-invertibility of the procedure: from the resampled image, even given the resampling grid, it is very computationally intensive and numerically unstable to reconstruct the original pseudo-raw data.

(ii) Re-appearance of the targets sidelobes: ideally, resampling a pseudo-raw image $u_{0}$ into $v_{0}$ using Algorithm 1 changes the oscillatory cardinal sine target profiles of $u_{0}$ (like the red-dashed line in Fig. 3) into discrete Diracs (like the blue-dotted line in Fig. 3). Unfortunately, if we need to manipulate at the subpixellic scale the resampled image $v_{0}$ for further processing, for instance for registration purpose, or simply if we try to oversample $v_{0}$ in order to retrieve the same pixel size as the initial image (recall the presence of zero-padding in Fig. 1), those discrete Diracs are interpolated as cardinal sines and we observe the re-appearance of their sidelobes, as in Fig. 7. This inability to manipulate the irregularly resampled image $v_{0}$ at the sub-pixellic scale restricts its potential use in image analysis algorithms requiring sub-pixellic precision.

(iii) Deterioration of the interferometric phase: when two (spatially registered) looks of the same scene are available, we are usually interested in computing the difference of their phases, since it is related to the elevation and motion of the scene. Unfortunately, irregularly resampling the two images independently introduces some decoherences between the images, and their interferometric phase is not well preserved by this procedure as we shall see in the next section.

In summary, irregularly resampling pseudo-raw images in order to minimize the sidelobes effects of their neighboring bright targets leads to images that are at the same time of high resolution, statisti-

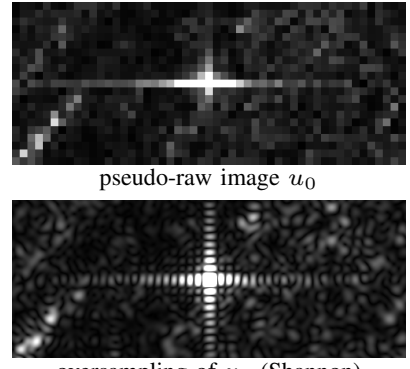

oversampling of $v_{0}$ (Shannon)

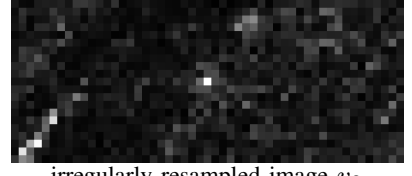

irregularly resampled image $v_{0}$

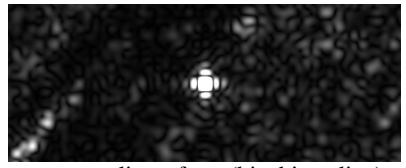

oversampling of $v_{0}$ (bicubic spline)
Fig. 7. Re-appearance of the targets sidelobes at the sub-pixellic scale. Thanks to the irregular resampling procedure, the targets induced cardinal sines present in the pseudo-raw image are changed into discrete Diracs in $v_{0}$. Therefore, the targets sidelobes present in $u_{0}$ are canceled in $v_{0}$. Unfortunately, we observe a re-appearance of the sidelobes when we oversample $v_{0}$ using the Shannon interpolation (see bottom-left image). Even when using a more local interpolation method to oversample $v_{0}$, such as bicubic splines (see bottom-right image), the sidelobes re-appear in the vicinity of the strong targets.

cally accurate, and well-suited for visual interpretation. However, this strategy performs poorly in terms of fidelity to the data, preservation of the interferometric phase, and leads to images that are not better suited than the initial pseudo-raw image for further processings requiring a sub-pixellic precision.

\section{SPECKLE Plus TARGETS DECOMPOSITION: A REVISITED CLEAN APPROACH}

In this section we explore the possibility to compute a decomposition of the image into two parts, one containing the bright targets (described by their sub-pixellic positions and complex amplitudes) and another one containing the image that would have been observed without the bright targets. This approach is not new and the most successful algorithm in that direction is the RELAX algorithm [25], which is an extension of the CLEAN algorithm that was first proposed in radio astronomy [22] before being used in microwave imaging [23]. The CLEAN approach consists in extracting one target at a time. The pixel with the brightest amplitude in the image is assumed to contain a target. The sub-pixellic position of the target is computed using maximum correlation (other criteria are possible). Once this subpixellic position is computed, the complex amplitude of the target is estimated using a least-square criterion. Then, the target is subtracted from the image and the algorithm is run again on the residual image. The RELAX approach is similar, except that each time a target is extracted from the data, the sub-pixellic positions and complex intensities of all previously detected targets are refitted according to a least-square criterion. These two algorithms can be viewed as matching pursuit algorithms, the dictionary being the infinite set of cardinal sines located at each possible point. Under that viewpoint, the CLEAN algorithm corresponds to the mere matching pursuit [37] while the RELAX algorithm is closer to the orthogonal matching pursuit [38].

In terms of performances, the CLEAN approach is not well suited for detecting multiple targets located near to each other, because it lacks a joint estimation of the sub-pixel locations of nearby targets. On the other hand, the RELAX approach is computationally intensive when the number of targets increases, since it requires to solve a sequence of non linear least squares problems with an increasing number of unknowns. This can be seen as a result of the high cardinality of the dictionary used in this orthogonal matching pursuit procedure. Is is important to note that both the CLEAN and RELAX approaches share the same weakness, they do not rely on a precise target detection criterion to decide whether a pixel of the image 
contains a strong target or not and the whole process does not rely on a satisfactory stopping criterion. Indeed, at each iteration, both algorithms assume that the pixel of the image with the brightest amplitude contains a target, the extraction process stops when the highest amplitude of the image falls below a given amplitude threshold, or after a given number of targets has been extracted from the image. In practice, the observed scene may exhibit a wide range of reflectivity values and we may observe numerous isolated bright targets whose amplitudes are large compared to the reflectivity of their surrounding areas (good signal-to-noise ratio), while being smaller or comparable to the amplitude of pure speckle in other areas. We illustrate this phenomenon on an airborne SAR image in Fig 8. A region delimited by a rectangle is magnified by Shannon interpolation. It contains a target with an amplitude $A_{0}$ that is more than ten times larger than that of the pixels in the surrounding area. The sidelobes of this target are thus visible, especially after reinterpolation. This target should thus be detected as such, even though the amplitude $A_{0}$ is below that of speckled areas with stronger reflectivity. The right part of Fig. 8 shows in red all pixels with an amplitude larger than $A_{0}$. Several pixels in the vegetated areas located at the left-hand side of the outlined target are marked in red as larger than $A_{0}$. More generally, numerous pixels marked in red do not exhibit a significantly larger amplitude than their surrounding neighbors and correspond to pure speckle (see the right-top side of the image). Consequently, a target extraction method with a stopping criterion based on the amplitude of the target, like CLEAN or RELAX, would either fail to detect the outlined target, or produce numerous false detections in pure speckle noise. The above mentioned limitation of CLEAN and RELAX can be addressed by using an explicit target detector in the extraction procedure.

Numerous approaches for target detection have been proposed in the literature (see e.g., [39], [40]). One of the most popular [41] is based on the generalized likelihood ratio test. The authors propose to detect various structures through the use of specially crafted masks (cross-shaped in the specific case of target detection) and the comparison of the mean intensity in the mask compared to a surrounding counterpart (cross excluded), using a so-called variation coefficient. However, variation coefficients are strongly dependent from the sampling since, as we showed earlier, the shape of a strong target may completely change according to the sampling grid. Taking this into account leads the authors to make a conservative choice yielding a sure detection at the cost of under-detection of less-brighter targets. This problem has lead to a multitude of CFAR type detectors like CA-CFAR, OS-CFAR, GO-CFAR and SO-CFAR, each of which being more effective in certain situations (multiple targets, targets in the vicinity of an edge, etc). In turn, some authors proposed a strategy to determine which of these detectors would be the more effective at any location of the image (see [39], [42]). We believe that resampling the image at sub-pixel scale is a necessary step for any method that seeks simplicity and to be the most generic. This is also supported by the success of our resampling scheme presented in Section III that proved efficient enough to suppress the sidelobes while being very simple.

In the rest of this section, we design a revisited CLEAN procedure that can be used to decompose the pseudo-raw image into a speckledominated component and a set of targets. Our revisited CLEAN procedure relies on an efficient sub-pixellic target detection criterion based on the so-called a contrario methodology [43], which leads to a well justified stopping criterion and an accurate control of the false alarms. This a contrario target detection criterion is presented in Section IV-A and the revisited CLEAN procedure is described and validated through synthetic and real-life experiments in Section IV-B. Last, several interesting applications of the decomposition provided

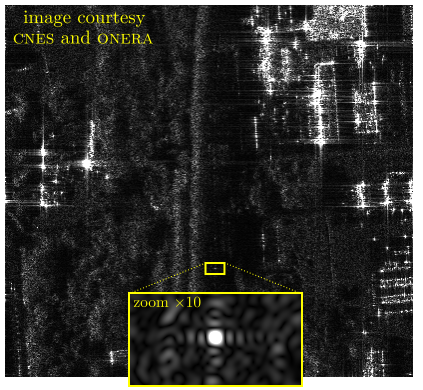

Airborne SAR pseudo-raw image, with a focus on a target with amplitude $A_{0}$

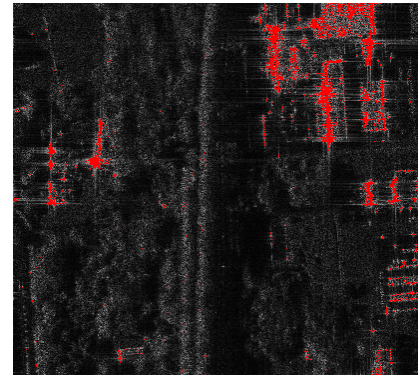

saturation (in red) of the pixels with amplitude higher than $A_{0}$
Fig. 8. Example of a bright target with an amplitude below the level of the speckle in high-reflectivity areas. (left) Airborne pseudo-raw image (copyright CNES and ONERA) with a zoom on a small region that contains a target of amplitude $A_{0}$; (right) pixels with an amplitude greater or equal to $A_{0}$ are marked in red. Setting the global amplitude threshold of CLEAN or RELAX smaller than $A_{0}$ would result in numerous target extractions in areas marked in red, including pure speckle noise.

by the revisited CLEAN algorithm are proposed in Section IV-C.

\section{A. A contrario detection of bright targets centers}

The a contrario methodology is a mathematical framework dedicated to the design of detectors providing a rigorous control of the number of false detections, that is, the average number of detections allowed in pure noise data. This methodology was first proposed in [44] for the detection of alignments in images, and then applied to many different tasks, such as estimation of the modes of a histogram [45], detection of shape elements [46], of vanishing points [47], contrasted edges [48], or line segments [49]. In remote sensing, it has been applied to the detection of subpixellic changes in satellite imagery [50] and feature matching in SAR imaging [51]. The a contrario framework can be viewed as a mathematical formulation of a visual perception principle, the socalled Helmholtz principle, that was first formulated in the context of image analysis by Lowe in [52]. This principle states that the human perceptual system perceives structures that are unlikely to appear by chance in a random configuration. In practice, the mathematical formulation of this principle relies on two main ingredients: a naive model (noted $\mathcal{H}_{0}$ ) that describes what would be pure random data, and a measurement function that characterizes the type of structures looked for. Given those two ingredients, the a contrario methodology can be applied to design a Number of False Alarms (NFA), which is a real-valued function that groups the structure according to their average number of appearances in $\mathcal{H}_{0}$. More precisely, the NFA is designed in such a way that, in pure random data $\mathcal{H}_{0}$, the expectation of the number of structures having a NFA smaller than $\varepsilon$ is less than $\varepsilon$. In practice, we detect the structures having a NFA smaller than a given threshold $\varepsilon$, those structures are said detectable at the level $\varepsilon$, or $\varepsilon$-meaningful, and this threshold parameter $\varepsilon$ has a handy meaning for the user since it simply represents an upper bound of the average number of (false) detections allowed in pure noise $\mathcal{H}_{0}$ (this result is formalized as the so-called NFA-property). The traditional setting $\varepsilon=1$ is often used to ensure that, in average, less than one detection is achieved in pure noise data. Compared to the classical statistical decision theory, the a contrario framework presents the advantage to get rid of the design of a $\mathcal{H}_{1}$ hypothesis, making the a contrario algorithm less sensitive to the modeling choice for the structures that we want to detect. Besides, this methodology yields algorithms having the NFA threshold $\varepsilon$ as the unique parameter, and the setting of this parameter is particularly simple thanks to the tangible interpretation of $\varepsilon$ offered by the NFA-property. We 
refer to [43] for a much more detailed presentation of the a contrario framework.

In the following, we propose to apply the a contrario methodology to the problem of the detection of strong targets in pseudo-raw images. First, we define the $\mathcal{H}_{0}$ model that describes the data when no target is to be detected.

Definition 1 ( $\mathcal{H}_{0}$ model). A random pseudo-raw image $\boldsymbol{u}_{\mathbf{0}}: \omega \rightarrow \mathbb{C}$ follows the $\mathcal{H}_{0}$ model $\left(\boldsymbol{u}_{0} \sim \mathcal{H}_{0}\right)$ if the sequences of its real and imaginary parts $\left\{\boldsymbol{u}_{\mathbf{0}}^{r}(k, \ell)\right\}_{(k, \ell) \in \omega}$ and $\left\{\boldsymbol{u}_{\mathbf{0}}^{i}(k, \ell)\right\}_{(k, \ell) \in \omega}$ are independent and identically distributed according to a standard normal distribution $\mathcal{N}(0,1)$.

The $\mathcal{H}_{0}$ model defined above corresponds to the case where $\boldsymbol{u}_{\mathbf{0}}$ is a pure speckle with a reflectivity equal to 2 . The choice of the unitary variances in Definition 1 is arbitrary and will have no incidence in the design of the NFA since the measurements that we are going to perform in $\mathcal{H}_{0}$ are independent from this choice.

Now, let us focus on the design of the measurement function that will be used in a probabilistic framework to compute the amount of surprise associated with the observation of a bright target in $\mathcal{H}_{0}$. Our underlying idea is very simple. A bright target is roughly characterized by the fact that its amplitude is large compared to the reflectivity of its surrounding area. Unfortunately, due to the sampling, the total amplitude of the target can be smeared in the vicinity of its center (this is the sidelobe effect) which makes difficult the estimation of the reflectivity of its surrounding area. However, this difficulty can be easily overcome as we explained in Section III how to compute the local displacement field $T=\left(T_{x}, T_{y}\right)$ : $\omega \rightarrow\left[-\frac{1}{2}, \frac{1}{2}\right] \times\left[-\frac{1}{2}, \frac{1}{2}\right]$ that best reduces the sidelobes. Indeed, if the pixel $\left(k_{0}, \ell_{0}\right)$ of $u_{0}$ contains the center of a strong target, then, the sub-pixellic position of the target center should be well approximated by $\left(k_{0}-T_{x}\left(k_{0}, \ell_{0}\right), \ell_{0}-T_{y}\left(k_{0}, \ell_{0}\right)\right)$. Therefore, the translated signal $(k, \ell) \mapsto U_{0}\left(k-T_{x}\left(k_{0}, \ell_{0}\right), \ell-T_{y}\left(k_{0}, \ell_{0}\right)\right)$ should behave as a discrete Dirac in the vicinity of $\left(k_{0}, \ell_{0}\right)$, so that we are able to efficiently compare the target amplitude to the reflectivity of its surrounding area. Based on this idea, we propose again to process the range and azimuth directions independently in order to reduce the computational costs by keeping all computations in the mono-dimensional case and avoiding the computation of all twodimensional translations with vector $\left(t_{x}, t_{y}\right) \in \mathbb{T} \times \mathbb{T}$. Besides, since we are only interested in the detection of the target centers, we can avoid the systematic computation of the local maxima $p_{0}(s)$ involved in (10), and assume that $p_{0}(s)=0$ which is also interesting (although not mandatory) in terms of reduction of the computational costs. This slight modification of the $\mathrm{TV}_{\text {mask }}^{\mathrm{d}}$ score (10) used in Algorithm 1 will be systematically assumed in the rest of this section.

Given a pseudo-raw image $u_{0}: \omega \rightarrow \mathbb{C}$, we compute the associated displacement field $T$ using Algorithm 1 with a given choice of $K$ and $N_{T}$ (we took $K=25$ and $N_{T}=20$ in all ours experiments). Let $\Delta \omega^{*}=[-K, K] \cap \mathbb{Z} \backslash\{0\}$ define a local neighborhood. First, we focus on the horizontal direction. For all $(k, \ell) \in \omega$, we define the quantity

$$
R_{x}^{u_{0}}(k, \ell)=\sqrt{\frac{U_{0}^{r}\left(k-T_{x}(k, \ell), \ell\right)^{2}}{\widehat{\sigma_{x}^{r}}(k, \ell)^{2}}+\frac{U_{0}^{i}\left(k-T_{x}(k, \ell), \ell\right)^{2}}{\widehat{\sigma_{x}^{i}}(k, \ell)^{2}}},
$$

where $\widehat{\sigma_{x}^{r}}(k, \ell)^{2}=\frac{1}{2 K} \sum_{k^{\prime} \in \Delta \omega^{*}} U_{0}^{r}\left(k-T_{x}(k, \ell)+k^{\prime}, \ell\right)^{2}$ is an empirical estimation of the variance of the horizontally translated signal $(k, \ell) \mapsto U_{0}^{r}\left(k-T_{x}(k, \ell), \ell\right)$ over the set $\left(k+\Delta \omega^{*}\right) \times\{\ell\}$, and $\widehat{\sigma_{x}^{i}}(k, \ell)^{2}$ is defined in the same way, simply by replacing the $U_{0}^{r}$ by $U_{0}^{i}$ in the definition of $\widehat{\sigma_{x}^{r}}(k, \ell)^{2}$. Again, if the image $u_{0}$ contains a bright target with a center located within the pixel $\left(k_{0}, \ell_{0}\right)$, the resampled signal $k \mapsto U_{0}\left(k-T_{x}\left(k_{0}, \ell_{0}\right), \ell_{0}\right)$ should behave as a discrete Dirac in the horizontal vicinity of $\left(k_{0}, \ell_{0}\right)$. Thus, the quantity $R_{x}^{u_{0}}\left(k_{0}, \ell_{0}\right)$ should efficiently measure the ratio between the target amplitude and the local reflectivity. Similarly, in the vertical direction, we set for all $(k, \ell) \in \omega$,

$$
R_{y}^{u_{0}}(k, \ell)=\sqrt{\frac{U_{0}^{r}\left(k, \ell-T_{y}(k, \ell)\right)^{2}}{\widehat{\sigma_{y}^{r}}(k, \ell)^{2}}+\frac{U_{0}^{i}\left(k, \ell-T_{y}(k, \ell)\right)^{2}}{\widehat{\sigma_{y}^{i}}(k, \ell)^{2}}},
$$

where $\widehat{\sigma_{y}^{r}}(k, \ell)^{2}=\frac{1}{2 K} \sum_{\ell^{\prime} \in \Delta \omega^{*}} U_{0}^{r}\left(k, \ell-T_{y}(k, \ell)+\ell^{\prime}\right)^{2}$ denotes this time an empirical estimation of the variance of the vertically translated signal $(k, \ell) \mapsto U_{0}^{r}\left(k, \ell-T_{y}(k, \ell)\right)$ over the set $\{k\} \times$ $\left(\ell+\Delta \omega^{*}\right)$, and again, $\widehat{\sigma_{y}^{i}}(k, \ell)^{2}$ is obtained by replacing $U_{0}^{r}$ by $U_{0}^{i}$ in the above definition of $\widehat{\sigma_{y}^{r}}(k, \ell)^{2}$. As measurement function for our a contrario model, we propose to compute, for all pseudo-raw image $u_{0}: \omega \rightarrow \mathbb{C}$, and all position $(k, \ell) \in \omega$, the quantity $R^{u_{0}}(k, \ell)$ defined by

$$
R^{u_{0}}(k, \ell)=\max \left(R_{x}^{u_{0}}(k, \ell), R_{y}^{u_{0}}(k, \ell)\right) .
$$

For the sake of completeness, the computation of (12) is explicitly described in Algorithm 2.

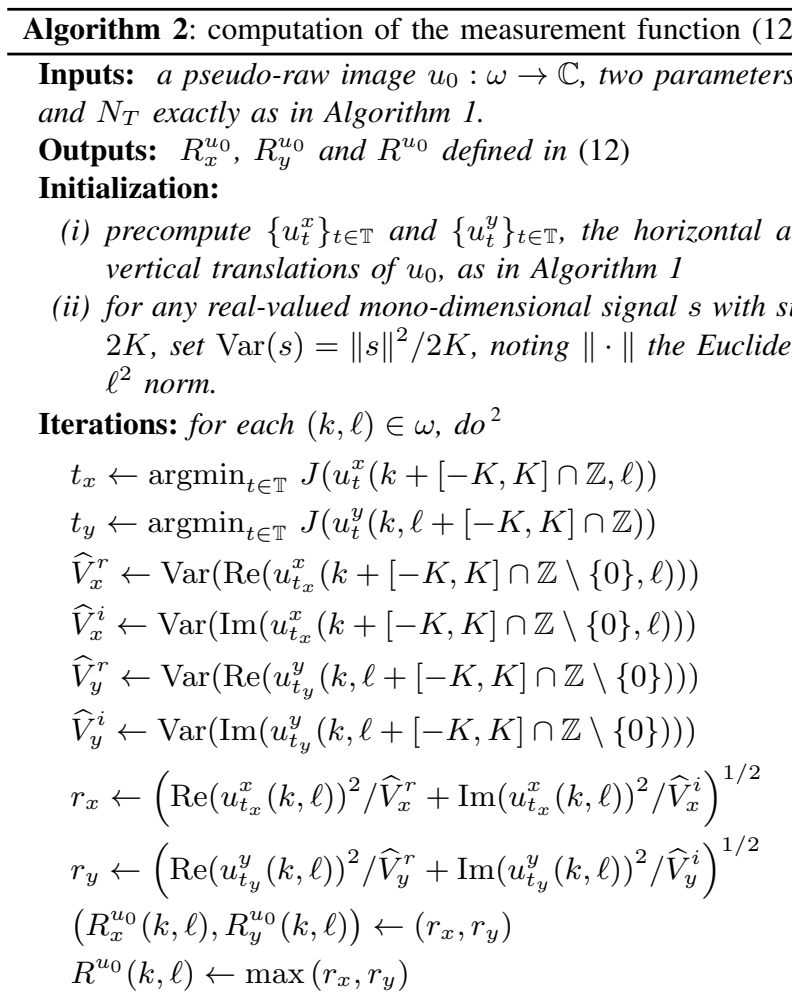

${ }^{2}$ We denote by Re and Im the real and imaginary part operators. Notice that in (13a)-(13f), a periodic convention is used for the images $u_{t}^{x}$ and $u_{t}^{y}$ when their evaluation outside of $\omega$ is needed. Optional: in (13a) and (13b) we can force $p_{0}(s)=0$ in the definition of $J$ (Eq. (10)) in order to save some computational time. All our experiments take into account this modification.

By construction, the measurement function $R^{u_{0}}$ should take large values at pixels $\left(k_{0}, \ell_{0}\right) \in \omega$ when a strong target is present in $u_{0}$ at the sub-pixellic position $\left(k_{0}-T_{x}\left(k_{0}, \ell_{0}\right), \ell_{0}-T_{y}\left(k_{0}, \ell_{0}\right)\right)$. However, instead of thresholding the measurements $\left\{R^{u_{0}}(k, \ell)\right\}_{(k, \ell) \in \omega}$ using an arbitrary threshold to decide for each pixel whether a target is present or not, the a contrario methodology suggests evaluating the amount of surprise associated with the observation in $\mathcal{H}_{0}$ (that is, when $u_{0}$ is a realization of $\boldsymbol{u}_{\mathbf{0}} \sim \mathcal{H}_{0}$ ) of the measurements $\left\{R^{u_{0}}(k, \ell)\right\}_{(k, \ell) \in \omega}$. The evaluation of such amount of surprise can 
be done using the complementary cumulative distribution functions $F_{k, \ell}$ defined by

$$
\forall(k, \ell) \in \omega, \forall r>0, \quad F_{k, \ell}(r)=\mathbb{P}_{\mathcal{H}_{0}}\left(R^{u_{0}}(k, \ell) \geq r\right)
$$

In order to evaluate the cumulative distribution functions $\left\{F_{k, \ell}\right\}_{(k, \ell) \in \omega}$ in the case $\boldsymbol{u}_{\mathbf{0}} \sim \mathcal{H}_{0}$, let us focus on the distribution of the random variables $\left\{R_{x}^{u_{0}}(k, \ell)\right\}_{(k, \ell) \in \omega}$ and $\left\{R_{y}^{u_{0}}(k, \ell)\right\}_{(k, \ell) \in \omega}$. In the simple case where we force the translation map to be constant over $\omega$ and the locality parameter $K$ to be large, the distribution of the random variables $R_{x}^{u_{0}}(k, \ell)$ and $R_{y}^{u_{0}}(k, \ell)$ is very well approached by a Rayleigh distribution, with a parameter $\sigma=1$ (since both quantities $R_{x}^{u_{0}}(k, \ell)$ and $R_{y}^{u_{0}}(k, \ell)$ can be viewed as the $\ell^{2}$ norm of a two-dimensional Student vector with $2 K$ degrees of freedom). When the displacement field $T$ is computed using Algorithm 1 over a random pseudo-raw image $\boldsymbol{u}_{\mathbf{0}} \sim \mathcal{H}_{0}$, we observed that the distribution of $R_{x}^{u_{0}}(k, \ell)$ and $R_{y}^{u_{0}}(k, \ell)$ remained well approached by a Rayleigh distribution, with a parameter $\sigma\left(K, N_{T}\right)$ that depends of the choice of $K$ and $N_{T}$ used in Algorithm 1. This observation is illustrated in Fig. 9, where we can see that the Rayleigh approximation seems even more accurate for large values of $K$, as can be observed by comparing Fig. 9 (a) to Fig. 9 (b) or Fig. 9 (c) to Fig. 9 (d). In particular, the choice $K=25$ leads to the empirical distributions displayed in Fig. 9 (b) and Fig. 9 (d) that are well approximated by a Rayleigh distribution, although they exhibit a slightly heavier tail than that of the actual Rayleigh distribution. This leads us to formulate the following assumption, on which our NFA-property relies.

Assumption 1. If $\boldsymbol{u}_{\mathbf{0}}$ follows the $\mathcal{H}_{0}$ model, then the sequences $\left\{R_{x}^{u_{0}}(k, \ell)\right\}_{(k, \ell) \in \omega}$ and $\left\{R_{y}^{u_{0}}(k, \ell)\right\}_{(k, \ell) \in \omega}$ are made of random variables that follow a Rayleigh distribution with parameter $\sigma\left(K, N_{T}\right)$ that depends on the values of $K$ and $N_{T}$ used in Algorithm 1 to compute the displacement field $T$.

Proposition 1 (NFA-property). Under Assumption 1, the real-valued function $\mathrm{NFA}: \mathbb{R}_{+} \rightarrow \mathbb{R}_{+}$defined by

$$
\forall r \geq 0, \quad \operatorname{NFA}(r)=2 \cdot|\omega| \cdot e^{-\frac{r^{2}}{2 \sigma\left(K, N_{T}\right)^{2}}}
$$

is a Number of False Alarms for the sequence of random measurements $\left\{R^{u_{0}}(k, \ell)\right\}_{(k, \ell) \in \omega}$ in the sense that it satisfies the NFAproperty, that is, for all $\varepsilon>0$,

$$
\mathbb{E}_{\boldsymbol{u}_{\mathbf{0}} \sim \mathcal{H}_{0}}\left[\#\left\{(k, \ell) \in \omega, \operatorname{NFA}\left(R^{\boldsymbol{u}_{\mathbf{0}}}(k, \ell)\right) \leq \varepsilon\right\}\right] \leq \varepsilon,
$$

where \#A denotes the cardinality of the set $A$.

Proof. Under Assumption 1, all measurements $\left\{R_{x}^{u_{0}}(k, \ell)\right\}_{(k, \ell) \in \omega}$ and $\left\{R_{y}^{u_{0}}(k, \ell)\right\}_{(k, \ell) \in \omega}$ follow a Rayleigh distribution with parameter $\sigma\left(K, N_{T}\right)$. Therefore, the complementary cumulative distribution functions $F_{k, \ell}$ can be bounded from above using

$$
\forall r>0, \forall(k, \ell) \in \omega, \quad F_{k, \ell}(r) \leq G(r):=2 \cdot e^{-\frac{r^{2}}{2 \sigma\left(k, N_{T}\right)^{2}}},
$$

simply because we have the relation

$$
F_{k, \ell}(r) \leq \mathbb{P}_{\mathcal{H}_{0}}\left(R_{x}^{u_{0}}(k, \ell) \geq r\right)+\mathbb{P}_{\mathcal{H}_{0}}\left(R_{y}^{u_{0}}(k, \ell) \geq r\right),
$$

and $\mathbb{P}_{\mathcal{H}_{0}}\left(R_{x}^{u_{0}}(k, \ell) \geq r\right)=\mathbb{P}_{\mathcal{H}_{0}}\left(R_{y}^{u_{0}}(k, \ell) \geq r\right)=e^{-\frac{r^{2}}{2 \sigma\left(k, N_{T}\right)^{2}}}$. Thus, the NFA-property (16) follows as a direct consequence of [53, Proposition 2]. However, let us finish this proof with a reformulation of this result for the sake of completeness. Let $\varepsilon>0$, let $E=\mathbb{E}_{\boldsymbol{u}_{\mathbf{0}} \sim \mathcal{H}_{0}}\left[\#\left\{(k, \ell) \in \omega, \operatorname{NFA}\left(R^{\boldsymbol{u}_{0}}(k, \ell)\right) \leq \varepsilon\right\}\right]$, and for

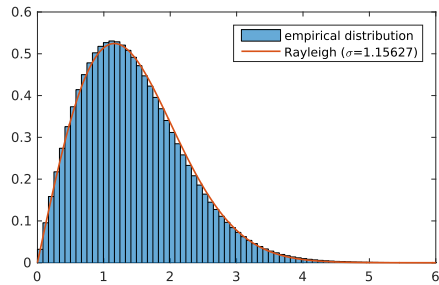

(a) $\mathrm{K}=10$ and $\mathrm{N}_{\mathrm{T}}=3$

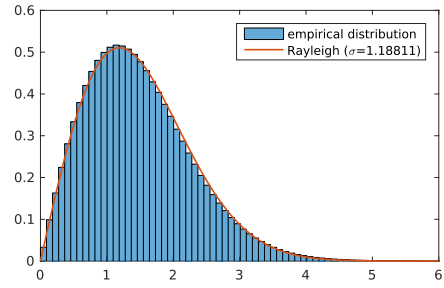

(c) $\mathrm{K}=10$ and $\mathrm{N}_{\mathrm{T}}=20$

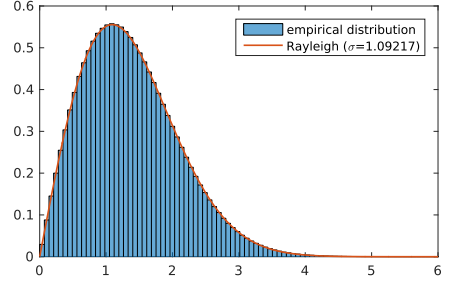

(b) $\mathrm{K}=25$ and $\mathrm{N}_{\mathrm{T}}=3$

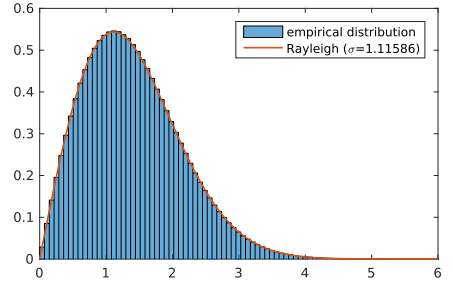

(d) $\mathrm{K}=25$ and $\mathrm{N}_{\mathrm{T}}=20$

Fig. 9. Distribution of $\left\{R_{x}^{u_{0}}(k, \ell)\right\}_{(k, \ell) \in \omega}$ in $\mathcal{H}_{0}$. Each graph represents the empirical histogram of the sequence $\left\{R_{x}^{u_{0}}(k, \ell)\right\}_{(k, \ell) \in \omega}$ (whose values are represented along the horizontal axis) computed from a $5000 \times 5000$ sized sample $u_{0}$ of $\boldsymbol{u}_{0} \sim \mathcal{H}_{0}$ using different settings of the parameters $K$ and $N_{T}$ in Algorithm 2. We can see that those histograms can be well fitted by some Rayleigh probability density functions of the type $x \mapsto\left(x / \sigma^{2}\right) \exp \left(-x^{2} /\left(2 \sigma^{2}\right)\right)$ whose parameter $\sigma$, estimated from $\left\{R_{x}^{u_{0}}(k, \ell)\right\}_{(k, \ell) \in \omega}$ using a maximum likelihood approach, depends on the setting of $\left(K, N_{T}\right)$. The same observations hold for the sequence $\left\{R_{y}^{u_{0}}(k, \ell)\right\}_{(k, \ell) \in \omega}$. Therefore, we can consider that Assumption 1 is approximately satisfied, although not rigorously exact.

each $(k, \ell) \in \omega$, let us denote by $\mathbb{1}_{\mathrm{NFA}\left(R^{\left.u_{0}(k, \ell)\right) \leq \varepsilon}\right.}$ the random variable defined by

$$
\mathbb{1}_{\mathrm{NFA}\left(R^{\left.u_{0}(k, \ell)\right) \leq \varepsilon}\right.}= \begin{cases}1 & \text { if } \operatorname{NFA}\left(R^{u_{\mathbf{0}}}(k, \ell)\right) \leq \varepsilon \\ 0 & \text { otherwise. }\end{cases}
$$

We have

$$
\begin{aligned}
E & =\mathbb{E}_{\boldsymbol{u}_{\mathbf{0}} \sim \mathcal{H}_{0}}\left[\sum_{(k, \ell) \in \omega} \mathbb{1}_{\mathrm{NFA}\left(R^{\left.\boldsymbol{u}_{0}(k, \ell)\right) \leq \varepsilon}\right]}\right. \\
& =\sum_{(k, \ell) \in \omega} \mathbb{P}_{\mathcal{H}_{0}}\left(\operatorname{NFA}\left(R^{\boldsymbol{u}_{\mathbf{0}}}(k, \ell) \leq \varepsilon\right)\right. \\
& =\sum_{(k, \ell) \in \omega}^{(k, \in)} \mathbb{P}_{\mathcal{H}_{0}}\left(G\left(R^{\boldsymbol{u}_{\mathbf{0}}}(k, \ell)\right) \leq \varepsilon /|\omega|\right) \\
& \leq \sum_{(k, \ell) \in \omega} \mathbb{P}_{\mathcal{H}_{0}}\left(F_{k, \ell}\left(R^{\boldsymbol{u}_{\mathbf{0}}}(k, \ell)\right) \leq \varepsilon /|\omega|\right),
\end{aligned}
$$

where the inequality is due to the relation (17). Besides, thanks to the p-value property (see [53, Lemma 1]), we have the upper-bound $\mathbb{P}_{\mathcal{H}_{0}}\left(F_{k, \ell}\left(R^{u_{0}}(k, \ell)\right) \leq \varepsilon /|\omega|\right) \leq \varepsilon /|\omega|$, from which follows the inequality $E \leq \sum_{(k, \ell) \in \omega} \varepsilon /|\omega| \leq \varepsilon$ announced in Proposition 1 .

In practice, we can compute the NFA defined in (15) provided we replace the $\sigma\left(K, N_{T}\right)$ by an estimation $\widehat{\sigma}\left(K, N_{T}\right)$ obtained from the computation of $R_{x}^{u_{0}}$ and $R_{y}^{u_{0}}$ over a sample $u_{0}$ of $\boldsymbol{u}_{0} \sim \mathcal{H}_{0}$, this boils down to Algorithm 3 which can be used to evaluate the function $(k, \ell) \mapsto \operatorname{NFA}\left(R^{u_{0}}(k, \ell)\right)$ given any image $u_{0}$ with domain $\omega$. Under this framework, the center of a strong target is detected at the level $\varepsilon$ inside the pixel $(k, \ell)$ of the image $u_{0}$ if and only if we have $\operatorname{NFA}\left(R^{u_{0}}(k, \ell)\right) \leq \varepsilon$, so that the detection of the target centers can be efficiently done by thresholding the NFAmap computed using Algorithm 3. The actual control of the average number of false detections (made in pure random data following $\mathcal{H}_{0}$ ) predicted by Proposition 1 is tested in Fig. 10. In this experiment, we study the effective control of the average number of (false) detections made in pure random data $\mathcal{H}_{0}$. For that purpose, we computed over one thousand samples $u_{0}$ of $\boldsymbol{u}_{\mathbf{0}} \sim \mathcal{H}_{0}$ (with domain 


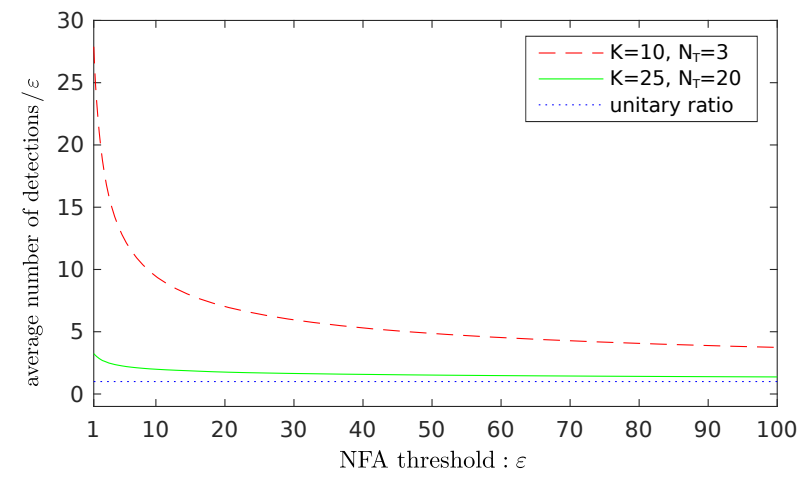

Fig. 10. Control of the average number of detections in pure $\mathcal{H}_{0}$ data. We display, using two curves (each curve represents a particular choice of the parameters $K$ and $N_{T}$ used in Algorithm 3), the evolution of the ratio between the average number of detections made in a pure speckle noise following $\mathcal{H}_{0}$ and $\varepsilon$, as a function of $\varepsilon$.

$\omega$ of size $1000 \times 1000)$ the average number of detections made at the level $\varepsilon$ (that is, the average number of positions $(k, \ell)$ such that $\left.\operatorname{NFA}\left(R^{u_{0}}(k, \ell)\right) \leq \varepsilon\right)$, for various values of $\varepsilon$. According to Proposition 1, this average number of detections should be less than $\varepsilon$, whatever the value of $\varepsilon$. In practice, we observe that it can be slightly higher, which is due to the imperfect approximation of the distribution of the random variables $\left\{R^{u_{0}}(k, \ell)\right\}_{(k, \ell) \in \omega}$ by a Rayleigh function (in particular for small values of $K$ ). Since Assumption 1 is not fully satisfied, the number of false detections is not rigorously controlled. However, since the number of detections remains comparable to its theoretical bound $\varepsilon$ (especially for large values of $K$ ), the control of the number of false detections remains satisfactory.

A synthetic example that illustrates the behavior of this detector of strong target centers is proposed in Fig. 11. In this experiment, we used a synthetic image with size $128 \times 512$ (see its modulus in Fig. 11 (a)) containing 466 targets with complex amplitudes of the type $\boldsymbol{A}=\rho \cdot e^{i \boldsymbol{\varphi}}$ with $\rho=10, \boldsymbol{\varphi} \sim \mathcal{U}_{[0,2 \pi]}$, and centers locations of the type $\left(\boldsymbol{x}_{\mathbf{0}}, \boldsymbol{y}_{\mathbf{0}}\right)=\left(k_{0}+\boldsymbol{t}_{\boldsymbol{x}}, \ell_{0}+\boldsymbol{t}_{\boldsymbol{y}}\right)$ where $\left(k_{0}, \ell_{0}\right)$ denotes a deterministic integer pixel position and $\left(\boldsymbol{t}_{\boldsymbol{x}}, \boldsymbol{t}_{\boldsymbol{y}}\right) \sim \mathcal{U}_{[-0.5,0.5] \times[-0.5,0.5]}$ a random translation. The synthetic image was also corrupted by a speckle noise with parameter $\sigma=1$ (that is, ten times less than the targets amplitudes, which is the typical configuration where the target sidelobes become visible in the vicinity of the target center of a pseudo-raw SAR image). The quality of the detection can be evaluated in terms of precision and recall. Those two quantities are

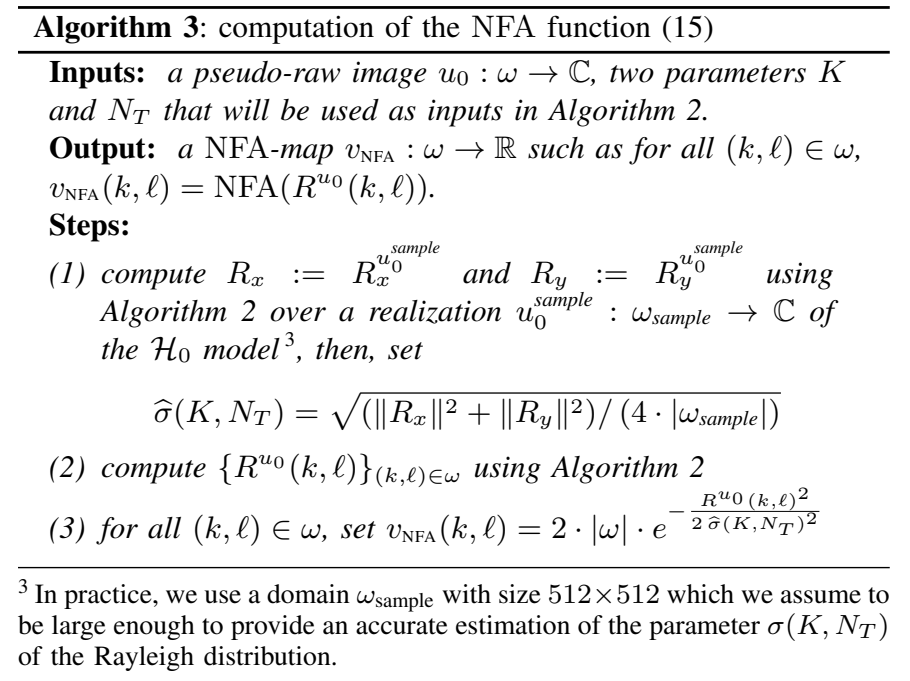

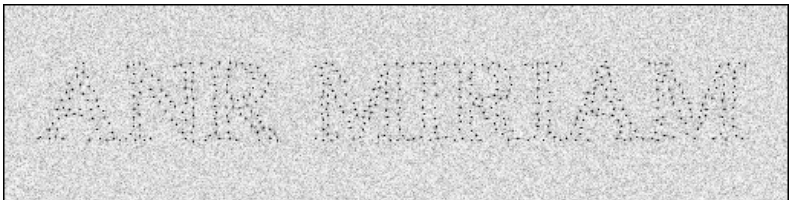

(a) input image (amplitude)

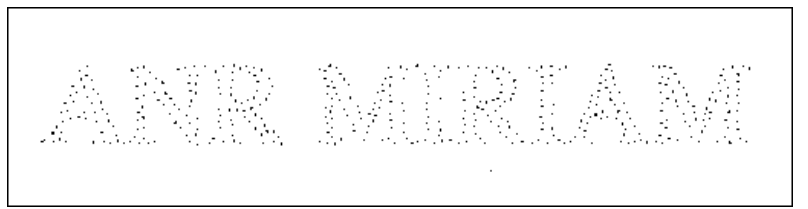

(b) NFA-threshold $\varepsilon=1$ : precision $=70 \%$, recall $=95 \%$

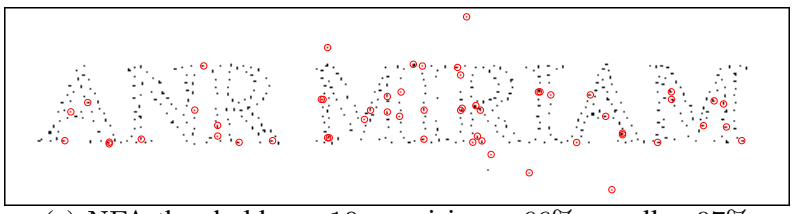

(c) NFA-threshold $\varepsilon=10$ : precision $=66 \%$, recall $=97 \%$ in red circled : differences between $(b)$ and $(c)$

Fig. 11. Target detection by NFA thresholding. A numerical simulation of strong targets mixed in stationary speckle is shown in (a). Targets are detected using Algorithm 3 with two threshold values: $\varepsilon=1$ (b) and $\varepsilon=$ 10 (c) corresponding to different trade-offs between a good precision (limited number of false alarms, setting of (b)) and high recall (few targets missed, setting of (c)).

defined by

$$
\text { precision }=\frac{\# \text { correct detections }}{\# \text { detected targets }}, \text { recall }=\frac{\# \text { correct detections }}{\# \text { targets }},
$$

so that 1 - precision represents the proportion of false positive detections among the set of all detected targets, and 1 - recall represents the proportion of false negative detections among the set of all actual targets. The detections results corresponding to $\varepsilon=1$ and $\varepsilon=10$ are displayed in Fig. 11 (b) and Fig. 11 (c). Since increasing $\varepsilon$ yields more detections, we display in black the detections common to both settings of $\varepsilon$, and in circled red the targets that were detected at the level $\varepsilon=10$ but not at the level $\varepsilon=1$. We can see that both settings yield a good recall score, which means that the targets centers are well detected. However, the precision score is worse than the recall due to an over-detection effect of targets centers around the true target centers, which will not be an issue for the applications discussed in the following paragraphs. We see that for $\varepsilon=1$ only one (false) detection is performed in the area with pure speckle (bottomcenter of the image), which is exactly what we expect according to Proposition 1. Increasing $\varepsilon$ improves the recall (thus, the number of correct detections) at the cost of increasing the false alarms in pure speckle, as seen in Fig. 11 (c).

\section{B. Speckle plus targets decomposition}

We derived in Section IV-A an a contrario criterion that can be used to decide whether or not the center of a strong target is present within a given pixel $\left(k_{0}, \ell_{0}\right) \in \omega$ of the pseudo-raw image $u_{0}$. Indeed, when $\operatorname{NFA}\left(R^{u_{0}}\left(k_{0}, \ell_{0}\right)\right) \leq \varepsilon$ (for instance, for $\varepsilon=1$ ), we assume that $u_{0}$ contains a strong target centered at the sub-pixellic position $\left(x_{0}, y_{0}\right):=\left(k_{0}-T_{x}\left(k_{0}, \ell_{0}\right), \ell_{0}-T_{y}\left(k_{0}, \ell_{0}\right)\right)$. Therefore, we can decompose $u_{0}$ into

$$
\forall(k, \ell) \in \omega, \quad u_{0}(k, \ell)=w_{0}(k, \ell)+A_{0} s_{0}(k, \ell),
$$

noting $A_{0} \in \mathbb{C}$ the complex amplitude of the target, $s_{0}(k, \ell)=$ $\operatorname{sinc}\left(k-x_{0}, \ell-y_{0}\right)$ and $w_{0}$ the image that would have been obtained in the absence of the target. In order to be able to extract the 
target from $u_{0}$ we need to compute an estimate $\widetilde{A}_{0}$ of the target amplitude $A_{0}$, which can be easily done by minimizing a leastsquares criterion, that is by computing

$$
\widetilde{A}_{0}=\underset{A_{0} \in \mathbb{C}}{\operatorname{argmin}}\left\|u_{0}-A_{0} s_{0}\right\|^{2}=\sum_{(k, \ell) \in \omega} u_{0}(k, \ell) s_{0}(k, \ell),
$$

as done for instance in [23]. One can remark that (19) also corresponds to the maximum likelihood estimator of $A_{0}$ if we assume in (18) that $w_{0}$ is a pure and stationary speckle (whatever its constant reflectivity). Finally, we can remove the target from $u_{0}$ by computing $u_{0}-\widetilde{A}_{0} s_{0}$ (which can be viewed as an estimate of $w_{0}$ in (18)) and repeat the process in a greedy way until no more targets are detected in the image.

Usually, the practical implementation of the a contrario detectors consists in extracting the $\varepsilon$-meaningful structures in decreasing NFA order. In our case, this would boil down to the following steps:

(i) compute $\left\{R^{u_{0}}(k, \ell)\right\}_{(k, \ell) \in \omega}$ and find the position $(k, \ell) \in \omega$ such as $\operatorname{NFA}\left(R^{u_{0}}(k, \ell)\right)$ is minimal

(ii) if $\operatorname{NFA}\left(R^{u_{0}}(k, \ell)\right) \leq \varepsilon$, consider that a target is present in $u_{0}$ at the sub-pixellic position $\left(k-T_{x}(k, \ell), \ell-T_{y}(k, \ell)\right)$, estimate its amplitude using (19), subtract the target from $u_{0}$, add the target features (the center position and the complex amplitude of the target) to the set of extracted targets, then go back to (i).

The algorithm then stops when no more position $(k, \ell) \in \omega$ fulfills $\operatorname{NFA}\left(R^{u 0}(k, \ell)\right) \leq \varepsilon$. Such an approach involves the complete recomputation of $\left\{R^{u_{0}}(k, \ell)\right\}_{(k, \ell) \in \omega}$ before each target extraction, yielding a typical execution time of 15 seconds per extraction for an image with size $|\omega|=1000 \times 1000$. Thus, in presence of several thousands of targets, the total execution time may exceed a day which can be problematic in many situations. For that reason, we decide to adopt a strategy similar to CLEAN, by processing all positions $(k, \ell)$ in decreasing order of $\left|u_{0}(k, \ell)\right|$. The crucial difference between our approach and the classical CLEAN algorithm is that our a contrario criterion (that is, the comparison of $\operatorname{NFA}\left(R^{u_{0}}(k, \ell)\right)$ to the threshold $\varepsilon$ ) is used to decide whether a target extraction should be done at position $u_{0}(k, \ell)$ or not. In general situations, this strategy avoids the recomputation of $R^{u_{0}}$ over all the domain $\omega$ and offers a nice reduction of the execution time (in practice, by a factor 15). Our revisited CLEAN algorithm is described in Algorithm 4. Each target extracted from a given pseudo-raw image using Algorithm 4 is represented by a 3 -uple $(x, y, A) \in \mathbb{R}^{2} \times \mathbb{C}$ such that $(x, y)$ denotes the (sub-pixellic) position of the target center and $A$ its complex amplitude. The algorithm returns the set $\mathscr{C}$ of all extracted targets and the image $w_{0}$ obtained by removing from $u_{0}$ all the targets of $\mathscr{C}$. By construction, one can recover $u_{0}$ from $w_{0}$ and $\mathscr{C}$ using

$$
\forall(k, \ell) \in \omega, u_{0}(k, \ell)=w_{0}(k, \ell)+\mathcal{S}_{0}(\mathscr{C})(k, \ell),
$$

where we have set

$$
\mathcal{S}_{0}(\mathscr{C})(k, \ell)=\sum_{(x, y, A) \in \mathscr{C}} A \operatorname{sinc}(k-x, \ell-y) .
$$

We discuss in Fig. 12 the quality of the decomposition as well as the ability of Algorithm 4 to extract the correct number of targets into synthetic data made of a linear combination of ten targets mixed to stationary speckle. We can see in Fig. 12 that the number of targets extracted using Algorithm 4 is comparable to $10+\varepsilon$ (ten correct detections and $\varepsilon$ false detections), excepting for large levels of noise that lead to less detections (the targets are not strong anymore compared to the noise level), or for small levels of noise in which case the number of detections increases due to the sensitiveness of the NFA model to the presence of residual sidelobes which remain in strong contradiction with the $\mathcal{H}_{0}$ model, leading to additional detections.

\begin{tabular}{cccc}
\hline $\begin{array}{c}\text { level of the } \\
\text { noise }(\sigma)\end{array}$ & $\begin{array}{c}\text { average number of } \\
\text { detected targets }\end{array}$ & MSE & PSNR (dB) \\
\hline 0.01 & 14.5 & $1.24 \cdot 10^{-6}$ & 59.1 \\
0.02 & 12.3 & $2.96 \cdot 10^{-6}$ & 55.3 \\
0.03 & 11.8 & $5.64 \cdot 10^{-6}$ & 52.5 \\
0.04 & 11.7 & $9.21 \cdot 10^{-6}$ & 50.4 \\
0.05 & 11.6 & $1.40 \cdot 10^{-5}$ & 48.5 \\
0.06 & 11.7 & $2.03 \cdot 10^{-5}$ & 46.9 \\
0.07 & 11.7 & $2.76 \cdot 10^{-5}$ & 45.6 \\
0.08 & 11.7 & $3.69 \cdot 10^{-5}$ & 44.3 \\
0.09 & 11.7 & $4.75 \cdot 10^{-5}$ & 43.2 \\
0.1 & 11.7 & $6.01 \cdot 10^{-5}$ & 42.2 \\
0.2 & 6.5 & $3.67 \cdot 10^{-4}$ & 34.4 \\
0.3 & 2.4 & $5.99 \cdot 10^{-4}$ & 32.2 \\
\hline
\end{tabular}

Fig. 12. Quality of the speckle plus target decomposition. We synthesized a set $\mathscr{C}_{0}$ containing ten targets $\left(x_{0}, y_{0}, A_{0}\right)$ with random center position $\left(x_{0}, y_{0}\right) \sim \mathcal{U}_{[0,100] \times[0,100]}$, and random complex amplitude $A_{0}=e^{i \varphi}$, such as $\varphi \sim \mathcal{U}_{[0,2 \pi]}$. The image $\mathcal{S}_{0}\left(\mathscr{C}_{0}\right)$ containing those targets was corrupted by a stationary speckle with reflectivity $2 \sigma^{2}$, by adding independently to the real and imaginary parts of $\mathcal{S}_{0}\left(\mathscr{C}_{0}\right)$ a Gaussian noise with zero-mean and standard deviation $\sigma$. Then, we used Algorithm 4 (with parameters setting $K=25, N_{T}=20$ and $\varepsilon=1$ ) to compute from the image the set of detected targets $\mathscr{C}$. The same experiment was repeated one thousand times for different noise level $\sigma$. On the second column of this Table, we display the average number of extracted targets, on the third column, the average mean square error (MSE) between $\mathcal{S}_{0}\left(\mathscr{C}_{0}\right)$ and $\mathcal{S}_{0}(\mathscr{C})$, and on the last column, the corresponding PSNR (using PSNR $=-10 \cdot \log _{10}(\mathrm{MSE})$ ).

Also, we display in Fig. 13 several examples of decompositions $u_{0}=w_{0}+\mathcal{S}_{0}(\mathscr{C})$ obtained from satellite and airborne images, or synthetic data.

\section{Algorithm 4: our revisited CLEAN algorithm \\ Inputs: a pseudo-raw image $u_{0}: \omega \rightarrow \mathbb{C}$, two parameters $K$ and $N_{T}$, and a threshold $\varepsilon>0$ for the NFA (15) that roughly represents the maximal allowed number of false detections. \\ Outputs: a cleaned image $w_{0}: \omega \rightarrow \mathbb{C}$ and a set of targets ${ }^{4} \mathscr{C}$. Initialization: \\ (i) compute $\widehat{\sigma}\left(K, N_{T}\right)$ as in step (1) of Algorithm 3 and set $\mathrm{NFA}: r \mapsto 2 \cdot|\omega| \cdot \exp \left(-\frac{r^{2}}{2 \widehat{\sigma}\left(K, N_{T}\right)^{2}}\right)$ \\ (ii) set $w_{0}=u_{0}, \mathscr{C}=\emptyset$ and $j=1$.}

Steps ${ }^{4}$ :

(1) compute $\left\{w_{t}^{x}\right\}_{t \in \mathbb{T}}$ and $\left\{w_{t}^{y}\right\}_{t \in \mathbb{T}}$ the horizontal and vertical translations of $w_{0}$ as in Algorithm 1

(2) if $(j \leq|\omega|)$, then find the position $\left(k_{j}, \ell_{j}\right) \in \omega$ of the $j^{\text {th }}$ brightest value of $\left|w_{0}\right|$, otherwise the algorithm stops

(3) compute $\left(t_{x}, t_{y}\right)=T\left(k_{j}, \ell_{j}\right)$ and $r=R^{w_{0}}\left(k_{j}, \ell_{j}\right)$

(4) if $\operatorname{NFA}(r)>\varepsilon$, then set $j=j+1$ and go back to (2)

(5) set $\mathscr{C}=\mathscr{C} \cup\{(x, y, A)\}$ where $x=k_{j}-t_{x}, y=\ell_{j}-t_{y}$ and $A=\sum_{(k, \ell) \in \omega} w_{0}(k, \ell) \operatorname{sinc}(k-x, \ell-y)$

(6) remove the target $(x, y, A)$ from $w_{0}$ by setting

$$
\forall(k, \ell) \in \omega, w_{0}(k, \ell)=w_{0}(k, \ell)-A \operatorname{sinc}(k-x, \ell-y)
$$

(7) set $j=1$ and go back to (1)

${ }^{4}$ Each target contained in $\mathscr{C}$ is characterized by a 3 -uple $(x, y, A)$ where $(x, y) \in \mathbb{R}^{2}$ denotes the coordinates of the target center, and $A \in \mathbb{C}$ its amplitude. ${ }^{1}$ Notice that step (3) amounts to apply to $w_{0}$ (instead of $u_{0}$ ) the steps (13a)-(13j) of Algorithm 2. Note also that, in step (5), the target amplitude $A$ can be efficiently computed as the inner product between the mono-dimensional signals $\ell \mapsto w_{t_{x}}^{x}\left(k_{j}, \ell\right)$ and $\ell \mapsto \operatorname{sinc}\left(\ell-\ell_{j}+t_{y}\right)$. 

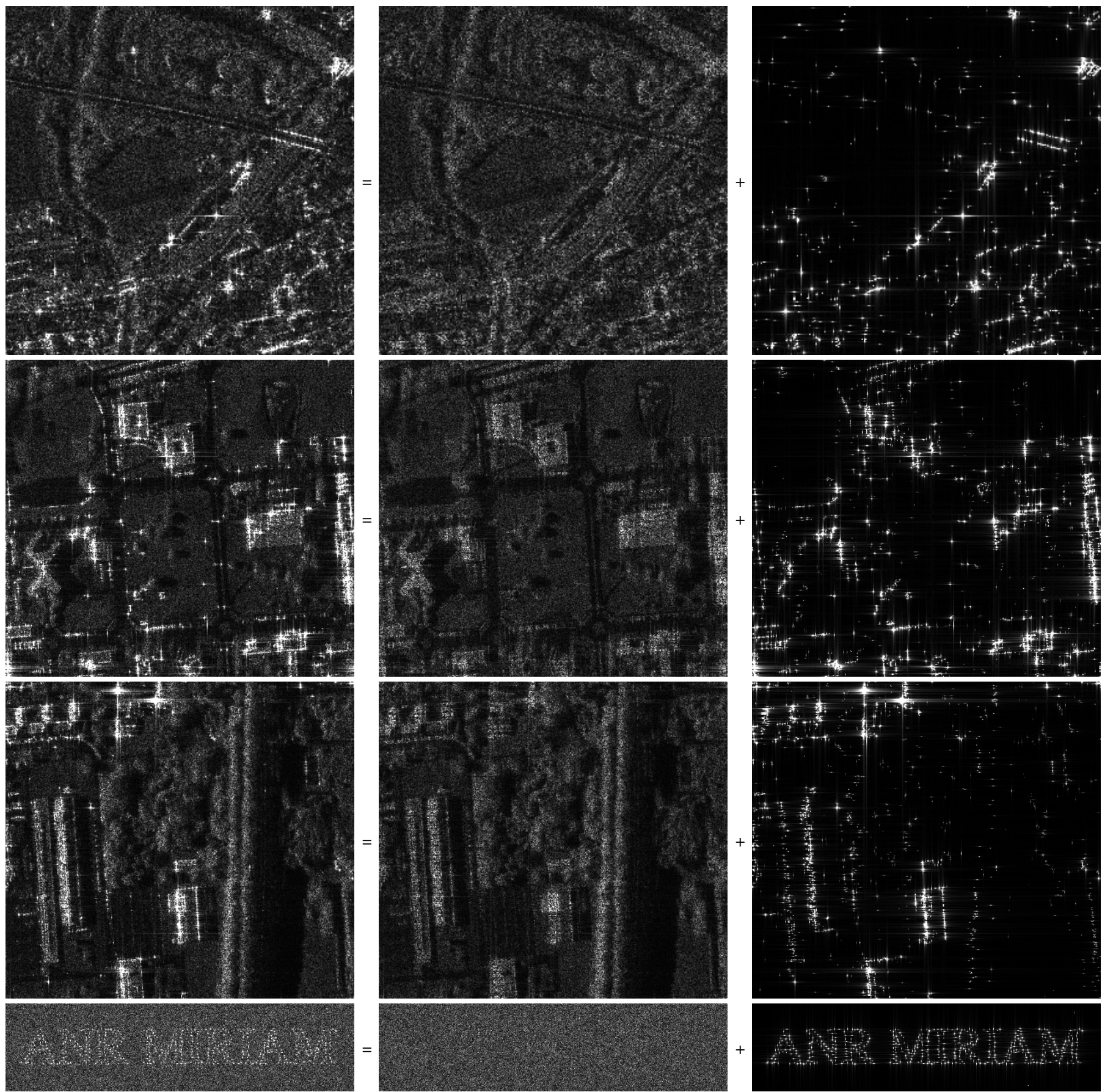

$u_{0}$

$w_{0}$

\section{$\mathcal{S}_{0}(\mathscr{C})$}

Fig. 13. Decomposition of SAR images into targets and background. After detection of targets in a SAR image $u_{0}$ (first column) using Algorithm 4, two images are computed: the residual image $w_{0}$ that contains the speckle-dominated background (center column) and the component $\mathcal{S}_{0}(\mathscr{C})($ see Eq. $(21))$ due to detected targets (third column). 


\section{Applications of the decomposition}

The extraction from a pseudo-raw image $u_{0}: \omega \rightarrow \mathbb{C}$ of a set of targets $\mathscr{C}$ offered by Algorithm 4 can be used in various interesting ways. First, it can be used to suppress the target sidelobes effect in the pseudo-raw image at arbitrary resolution. Indeed, since the image $w_{0}$ produced by Algorithm 4 is free of targets, it can be efficiently resampled (for instance, using the Shannon interpolation) at any resolution. Once image $w_{0}$ is resampled on the new sampling domain, we can add the targets without creating sidelobes effect simply by changing the coordinates of all target centers to make them coincide with the new sampling grid. Let us describe more precisely how such manipulation can be done. Recall that the initial sampling domain $\omega=\{0, \ldots, m-1\} \times\{0, \ldots, n-1\}$ corresponds to the sampling with unitary step (in both range and azimuth directions) of the continuous spatial domain $\omega_{c}:=[0, m) \times[0, n)$. Let $\omega^{\prime}$ denote a finite subset of $\omega_{c}$ that represents the targeted sampling domain. For instance, one can take

$$
\omega^{\prime}=\frac{1}{z} \cdot\{0, \ldots, z \cdot m-1\} \times \frac{1}{z} \cdot\{0, \ldots, z \cdot n-1\}
$$

to perform a zooming $(z \geq 1)$ or unzooming $(z<1)$ with factor $z$ in both directions, but any other choice of $\omega^{\prime}$ (even irregular) can be considered. Let $\mathscr{D}_{\omega^{\prime}}(\mathscr{C}): \omega^{\prime} \rightarrow \mathbb{C}$ the recombination of $\mathscr{C}$ into discrete Diracs on the grid $\omega^{\prime}$, which is defined by

$$
\forall\left(x^{\prime}, y^{\prime}\right) \in \omega^{\prime}, \mathscr{D}_{\omega^{\prime}}(\mathscr{C})\left(x^{\prime}, y^{\prime}\right)=\sum_{(x, y, A) \in \mathscr{C}} A \delta_{\pi_{\omega^{\prime}}(x, y)}\left(x^{\prime}, y^{\prime}\right),
$$

where $\pi_{\omega^{\prime}}(x, y)=\operatorname{argmin}_{\left(x^{\prime}, y^{\prime}\right) \in \omega^{\prime}}\left\|\left(x-x^{\prime}, y-y^{\prime}\right)\right\|$ denotes a projection of $(x, y)$ over $\omega^{\prime}$, and $\delta_{\pi_{\omega^{\prime}}(x, y)}\left(x^{\prime}, y^{\prime}\right)$ is defined by

$\forall\left(x^{\prime}, y^{\prime}\right) \in \omega^{\prime}, \quad \delta_{\pi_{\omega^{\prime}}(x, y)}\left(x^{\prime}, y^{\prime}\right)= \begin{cases}1 & \text { if }\left(x^{\prime}, y^{\prime}\right)=\pi_{\omega^{\prime}}(x, y) \\ 0 & \text { otherwise, }\end{cases}$

so that $\delta_{\pi_{\omega^{\prime}}(x, y)}$ simply represents a discrete Dirac centered at the position $\pi_{\omega^{\prime}}(x, y) \in \omega^{\prime}$. Consequently, the image $\mathscr{D}_{\omega^{\prime}}(\mathscr{C})$ defined in (22) is a linear combination of discrete Diracs centered at different positions of the grid $\omega^{\prime}$, and does not present any sidelobes. Finally, one can resample $u_{0}$ over $\omega^{\prime}$ without producing undesirable sidelobe effects by computing $R_{\omega^{\prime}}\left(u_{0}\right): \omega^{\prime} \rightarrow \mathbb{C}$, defined by

$$
\forall\left(x^{\prime}, y^{\prime}\right) \in \omega^{\prime}, \quad R_{\omega^{\prime}}\left(u_{0}\right)\left(x^{\prime}, y^{\prime}\right)=W_{0}\left(x^{\prime}, y^{\prime}\right)+\mathscr{D}_{\omega^{\prime}}(\mathscr{C}),
$$

where $w_{0}$ and $\mathscr{C}$ denote the outputs of Algorithm 4 applied to $u_{0}$, and $W_{0}$ the Shannon interpolate of $w_{0}$.

A straightforward and interesting application consists in taking $\omega^{\prime}=\omega$ in (23), which exactly amounts to replace $\mathcal{S}_{0}(\mathscr{C})$ by $\mathscr{D}_{\omega}(\mathscr{C})$ in (20), and yields an image $R_{\omega}\left(u_{0}\right)$ with the same resolution as the initial pseudo-raw image $u_{0}$, but without undesirable sidelobes effects since all the detected strong targets are replaced by discrete Diracs, as illustrated in Fig. 14. Therefore, the computation of $R_{\omega}\left(u_{0}\right)$ can be considered as an alternative to the irregular resampling strategy presented in Section III, that leads to a similar sidelobes suppression while better preserving the pseudo-raw image in areas where no strong target is present. This advantage is particularly interesting to perform interferometry, as we shall discuss now.

As stated before, information about the elevation of a scene can be computed given two registered views of the same scene, provided we apply an appropriate ramp-phase to one of the two images in order to get rid of orbital fringes patterns. Noting $\Phi$ the operator corresponding to this transformation, and $u$ and $u^{\prime}$ the two registered SLC images, we can compute the coherence map as the quantity

$$
c\left(u, u^{\prime}\right)=\frac{\mu\left(u \cdot \overline{\Phi\left(u^{\prime}\right)}\right)}{\sqrt{\mu\left(|u|^{2}\right)} \cdot \sqrt{\mu\left(\left|\Phi\left(u^{\prime}\right)\right|^{2}\right)}},
$$
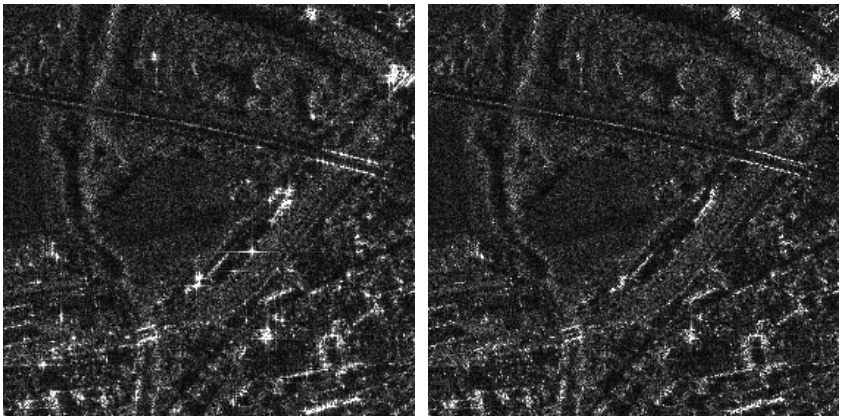

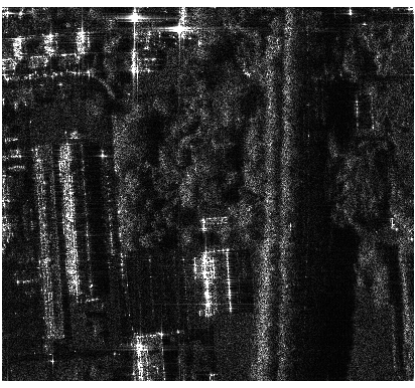

pseudo-raw image $u_{0}$

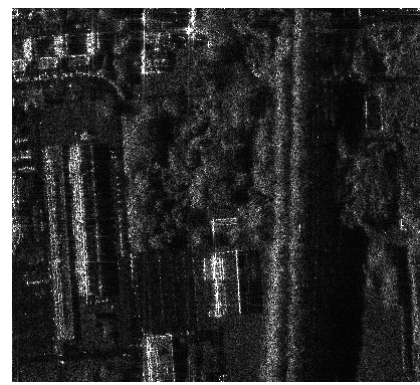

image $R_{\omega}\left(u_{0}\right)$
Fig. 14. Recombination of targets into discrete Diracs for sidelobes suppression. We used Algorithm 4 to compute from two pseudo-raw images $u_{0}$ (first column) a cleaned image $w_{0}$ and a set of targets $\mathscr{C}$. According to (20), each pseudo-raw image can be decomposed as $u_{0}=w_{0}+\mathcal{S}_{0}(\mathscr{C})$ where $\mathcal{S}_{0}(\mathscr{C})$ is made of a linear combination of cardinal sine functions sampled over $\omega$, corresponding to the contribution to $u_{0}$ of the targets present in $\mathscr{C}$ (see Fig. 13). By computing $R_{\omega}\left(u_{0}\right)$, using (23) with $\omega^{\prime}=\omega$, we replace the $\mathcal{S}_{0}(\mathscr{C})$ component by a linear combination of discrete Diracs on the grid $\omega$, leading to the images without sidelobes effects (second column).

where $\mu$ denotes a local averaging operator (the averaging domain used in our experiments was a disk with radius 2.5 pixels). The coherence map defined in (24) is designed to be high in areas grouping pixels with similar elevation, provided no decorrelation occurred between $u$ and $u^{\prime}$. In Fig. 15, we compare the coherence maps computed from apodized pairs of images (see Fig. 15 (a)), from pseudo-raw images (see Fig. 15 (b)), from irregular resamplings of those pseudo-raw images (see Fig. 15 (c)), or from the images obtained by applying the $R_{\omega}$ operator to the pseudo-raw images (see Fig. 15 (d)). Comparing Fig. 15 (a) to Fig. 15 (b) yields mitigated conclusions. On the one hand, apodization is responsible for overestimated coherence in Fig. 15 (a). On the other hand, over-coherent values can also be found in Fig. 15 (b) along the sidelobes of the strong targets (see the cross shape on the left side of Fig. 15 (b)). As can be seen in Fig. 15 (c), the irregular resampling strategy presented in Section III successfully solves the issue of over-coherence along the sidelobes of the strong targets, unfortunately it also induces a severe loss of coherence in areas where no strong target is present, due to the fact that the irregular grid used to resample both images may differ in those areas. The coherence map computed from $R_{\omega}\left(u_{0}\right)$ and $R_{\omega}\left(u_{0}^{\prime}\right)$, displayed in Fig. 15 (d), is clearly better since it also avoids over-coherence along sidelobes, but without introducing a global decoherence between the two images. This empirical conclusion is also confirmed in Fig. 16 using two coherent pseudo-raw SLC SAR images $u_{0}$ and $u_{1}$ made of synthetic sets of coherent targets $\mathscr{C}^{\star}$ and $\mathscr{C}_{1}^{\star}$ and coherent synthetic speckle $w_{0}^{\star}$ and $w_{1}^{\star}$. Indeed, we see that the over-coherence due to the target sidelobes in $c\left(u_{0}, u_{1}\right)$ (first row of Fig. 16) is avoided in $c\left(R_{\omega}\left(u_{0}\right), R_{\omega}\left(u_{1}\right)\right)$ (second row of Fig. 16). Besides, we see that the coherence of the background (due to the coherence of the synthetic speckle that we used) is also well preserved by the $R_{\omega}$ operator. Besides, we see that the coherence 


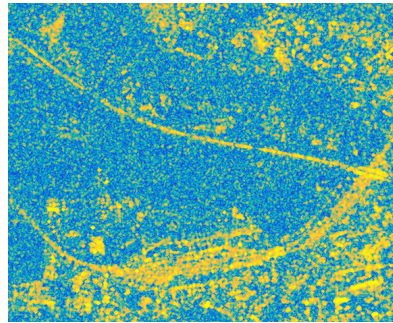

(a) apodized data
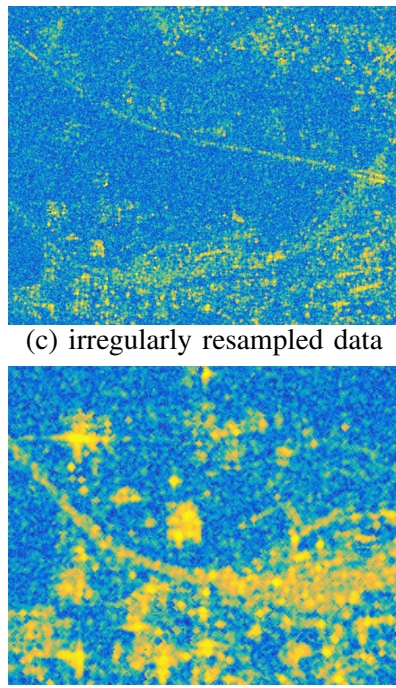

close-up view of (b) (c) irregularly resampled data

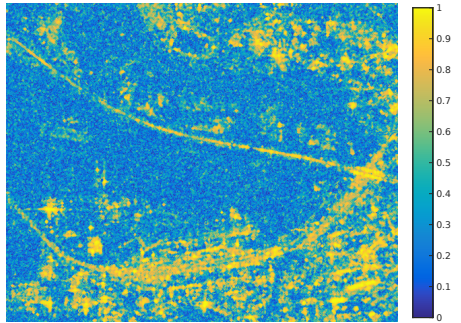

(b) pseudo-raw data

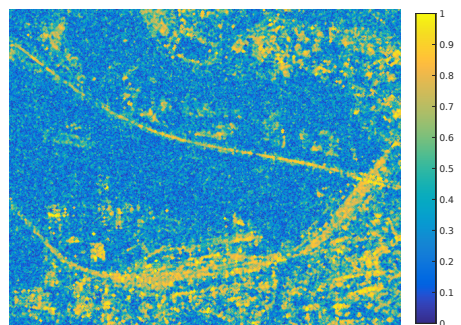

(d) $R_{\omega}$-processed data

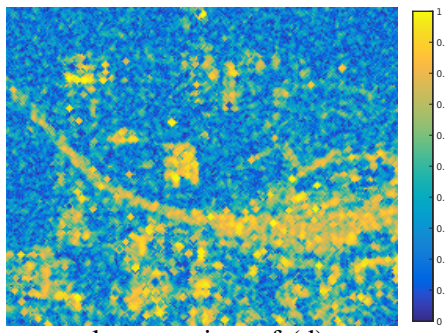

close-up view of (d)
Fig. 15. Coherence map of the interferometric phase. We display in (a) the coherence map $c\left(u_{\omega}, u_{\omega}^{\prime}\right)$ (Eq. (24)) computed from two registered TerraSAR-X SLC images undergoing apodization (but with no zero-padding). Removing the apodization yields two pseudo-raw images whose coherence map $c\left(u_{0}, u_{0}^{\prime}\right)$ is displayed in (b). We display in (c) the coherence map $c\left(v_{0}, v_{0}^{\prime}\right)$ computed from the irregular resamplings of $u_{0}$ and $u_{0}^{\prime}$, while we display in (d) that computed from $R_{\omega}\left(u_{0}\right)$ and $R_{\omega}\left(u_{0}^{\prime}\right)$.

map $c\left(R_{\omega}\left(u_{0}\right), R_{\omega}\left(u_{1}\right)\right)$ is very close to that we obtain by applying the $R_{\omega}$ operator to the actual ground-truth decomposition (third row of Fig. 16) showing that the $R_{\omega}$ operator successfully improves the coherence map in the vicinity of the centers of the strong targets thanks to the removal of the targets sidelobes, while preserving at the same time the coherence of the background.

The last experiment that we shall consider focuses on the joint resampling of a pseudo-raw image $u_{0}: \omega \rightarrow \mathbb{C}$ over a grid $\omega^{\prime} \neq \omega$ and the suppression of the sidelobes due to strong targets on this new resampling grid. As we already illustrated in Fig. 7, the irregular resampling procedure is not compatible with posterior sub-pixellic manipulations due to the re-appearance of sidelobes at sub-pixel scales. However, the $R_{\omega^{\prime}}$ operator can successfully achieve this task, as we illustrate in Fig. 17, where we have set

$$
\omega^{\prime}=\frac{m}{M}\{0,1, \ldots, M-1\} \times \frac{n}{N}\{0,1, \ldots, N-1\}
$$

and used $R_{\omega^{\prime}}$ to resample a full sequence of twenty-six pseudo-raw Terra-SAR-X images back to the initial grid used by the data-provider (recall that $(M, N)$ and $(m, n)$ are the image dimensions before and after resampling the image at the critical Nyquist rate, as illustrated in Fig. 1).

\section{CONCLUSION AND PERSPECTIVES}

In this paper, we addressed in two different ways the issue of sidelobes suppression with no loss of resolution and statistics preservation for pseudo-raw images. The first proposed approach consists

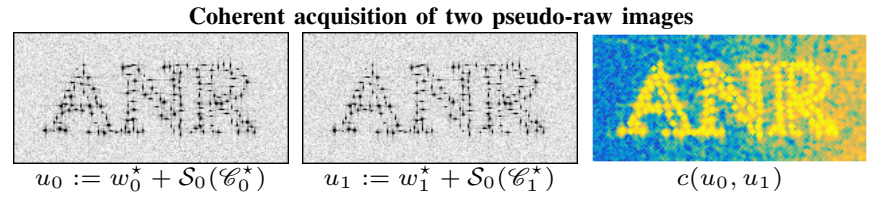

Estimated recombination with discrete Diracs

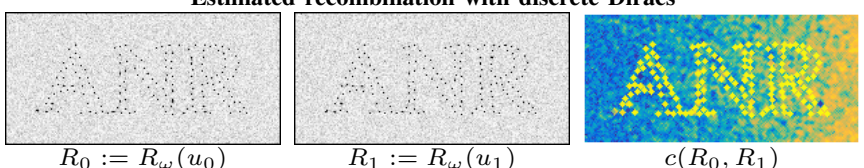

Ground-truth recombination with discrete Diracs

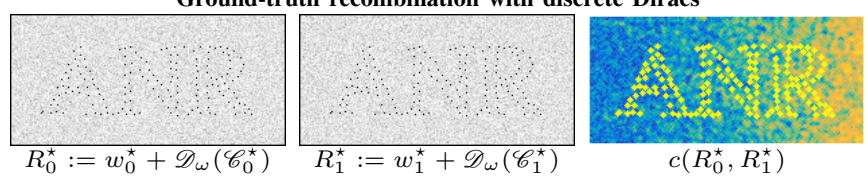

Fig. 16. Coherence map on synthetic coherent pseudo-raw acquisitions. The complex images $u_{0}$ and $u_{1}$, whose moduli are displayed in the first row, represent two synthetic coherent pseudo-raw images. Those images were synthesized using two coherent sets of targets $\mathscr{C}_{0}^{\star}$ and $\mathscr{C}_{1}^{\star}$ (each target in $\mathscr{C}_{0}^{\star}$ is also present in $\mathscr{C}_{1}^{\star}$ with the same complex amplitude but with a random shift of at most 0.1 pixel of its center position) and two coherent speckle images, noted $w_{0}^{\star}$ and $w_{1}^{\star}$, whose coherence is linearly increasing from $0 \%$ to $80 \%$ along the horizontal axis. Applying the $R_{\omega}$ operator to $u_{0}$ and $u_{1}$ yields the images $R_{0}$ and $R_{1}$, whose moduli are displayed on the second row. They can be compared to the images $R_{0}^{\star}$ and $R_{1}^{\star}$ that one would obtain if the speckle plus target decomposition was perfectly done. The coherence maps computed from the pair of images $\left(u_{0}, u_{1}\right),\left(R_{0}, R_{1}\right)$ and $\left(R_{0}^{\star}, R_{1}^{\star}\right)$ are displayed in the third column and share the same color scale as in Fig. 15.

in resampling the pseudo-raw image over an irregular grid that efficiently cancels the sidelobes in the vicinity of the strong targets while preserving the speckle statistics (in particular the valuable spatial uncorrelation of the speckle in the pseudo-raw images) in fully developed speckle areas. The irregular resampling grid as well as the resampled image are computed using a simple algorithm that exhibits a linear complexity with respect to the image size. However, the resampled images delivered by this scheme cannot be efficiently interpolated (due to the reappearance of the targets sidelobes at the sub-pixel scale) and are not well suited to applications where the preservation of the interferometric phase is needed.

The second approach that we proposed allows for the decomposition of the pseudo-raw image into a speckle-dominated component and a set of targets, using a revisited CLEAN algorithm that relies on a new detector of the sub-pixellic position of strong target centers specifically designed for pseudo-raw images. The latter detector is based on the a contrario methodology which provides an explicit control of the number of false detections as well as a well justified stopping criterion for the CLEAN procedure. The derived revisited CLEAN algorithm exhibits a higher computational complexity than the resampling algorithm (its complexity is proportional to the size of the image multiplied by the number of targets found in the image). However, the decomposition of the image that it provides is particularly suited to numerous SAR applications. In particular, it avoids the limitations related to the resampling strategy mentioned above. Indeed, we showed how the extracted targets could be recombined without creating sidelobes at any resolution. Besides, the decomposition is conservative (the initial pseudo-raw image can be recovered exactly from the two components of the decomposition), and we showed that it allows the efficient preservation, and even the improvement, of the interferometric phase in situations where several coherent views of the scene are available.

More generally, we believe that the ability to efficiently compute the decomposition using our revisited CLEAN algorithm opens very 

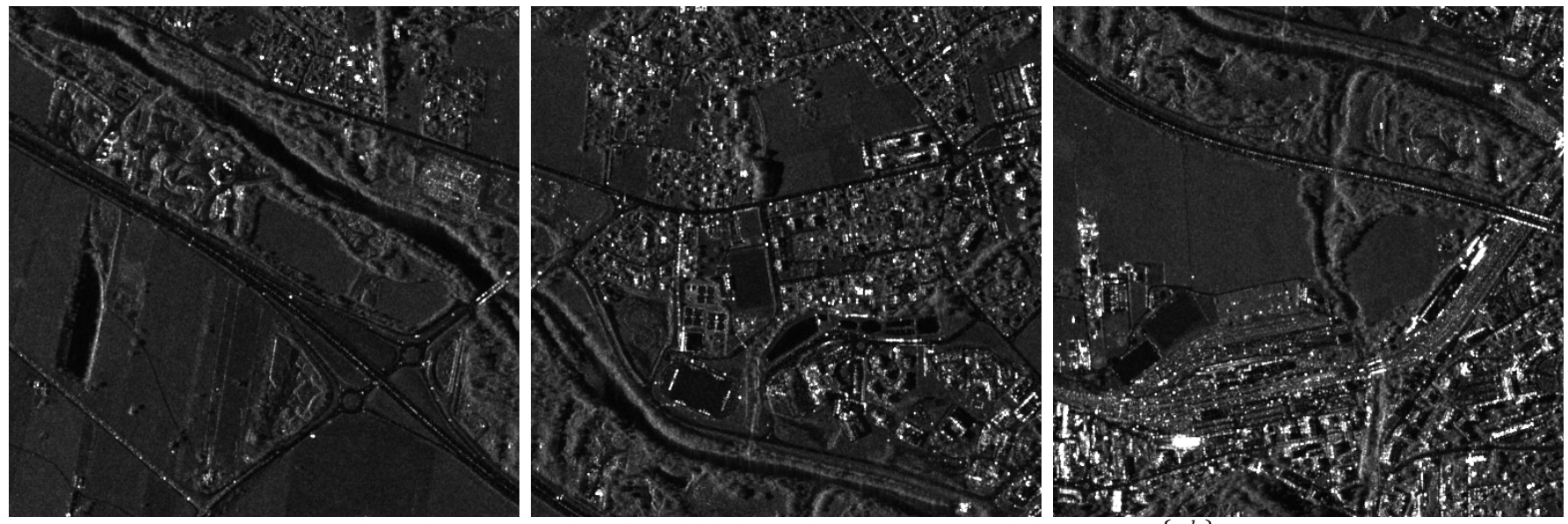

Temporal multi-look averaging of the initial (apodized and oversampled) sequence $\left\{u^{k}\right\}_{1 \leq k \leq 26}$
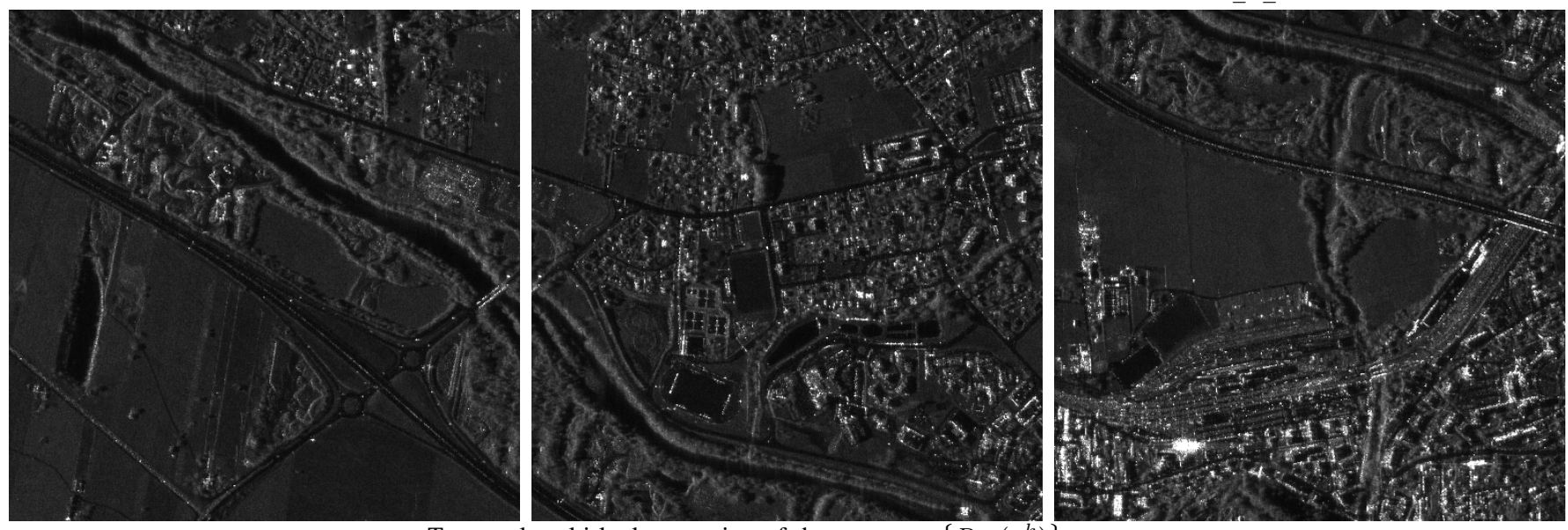

Temporal multi-look averaging of the sequence $\left\{R_{\omega^{\prime}}\left(u_{0}^{k}\right)\right\}_{1 \leq k \leq 26}$

Fig. 17. Temporal multi-look averaging at the initial resolution. First row: several parts of the temporal multi-look averaging computed from twenty-six TerraSAR-X images $\left\{u^{k}\right\}_{1 \leq k \leq 26}$ (used as provided by the spatial agency, i.e., undergoing apodization and oversampling with factors $M / m \approx 1.10$ and $N / n \approx 1.28$ in the range and azimuth directions). Second row: the temporal multi-look averaging of the sequence $\left\{R_{\omega^{\prime}}\left(u_{0}^{k}\right)\right\}_{1 \leq k \leq 26}$, where $\left\{u_{0}^{k}\right\}_{1 \leq k \leq 26}$ denotes the pseudo-raw sequence (with spatial domain $\omega$ of size $m \times n$ ) associated to $\left\{u^{k}\right\}_{1 \leq k \leq 26}$, and $\omega^{\prime}$ is defined in (25). We can see that using $R_{\omega^{\prime}}$ yields an efficient resampling of the pseudo-raw sequence since it benefits from the improved level of details of the pseudo-raw sequence (due to the absence of apodization) without suffering from undesirable sidelobe effects in the vicinity of the strong targets.

interesting perspectives since it splits the image into two components having strongly different natures. Indeed, in many situations, those two components are difficult to process when mixed together while processing the two components separately may drastically simplify the study. For instance, we can decide to focus only on the target component to perform detection tasks (alignments, clusters, changes, permanent scatterers, ...), feature matching, or correspondences between several looks (for instance for registration purpose). On the contrary, we can decide to only focus on the speckle-dominated component to perform tasks such as denoising, segmentation, classification, which may reveal more efficient in the absence of targets. It would be also interesting to investigate the possibility to design SAR image processing models that would rely on the two components jointly.

\section{SOURCE CODES}

Source codes corresponding to the algorithms described in this paper are freely available on the web pages of the authors.

\section{ACKNOWLEDGEMENTS}

We would like to thank the anonymous reviewers for their valuable suggestions. This work is supported by the ANR MIRIAM project ANR-14-CE27-0019.

\section{REFERENCES}

[1] F. Argenti, A. Lapini, T. Bianchi, and L. Alparone, "A tutorial on speckle reduction in synthetic aperture radar images," IEEE Geoscience and remote sensing magazine, vol. 1, no. 3, pp. 6-35, 2013.

[2] C.-A. Deledalle, L. Denis, F. Tupin, A. Reigber, and M. Jäger, "NL-SAR: a unified Non-Local framework for resolution-preserving (Pol)(In)SAR denoising," IEEE Transactions on Geoscience and Remote Sensing, vol. 53, no. 4, 2015.

[3] A. Lapini, T. Bianchi, F. Argenti, and L. Alparone, "A whitening method for the despeckling of SAR images affected by correlated speckle noise," in Proceedings of the 20th European Signal Processing Conference (EUSIPCO). IEEE, 2012, pp. 2487-2491.

[4] J. Aelterman, B. Goossens, A. Pizurica, and W. Philips, "Suppression of correlated noise," in Recent advances in signal processing. In-Tech, 2010, pp. 211-236.

[5] H. C. Stankwitz, R. J. Dallaire, and J. R. Fienup, "Nonlinear apodization for sidelobe control in SAR imagery," IEEE Transactions on Aerospace and Electronic Systems, vol. 31, no. 1, pp. 267-279, Jan 1995.

[6] X. Xu and R. M. Narayanan, "Enhanced resolution in SAR/ISAR imaging using iterative sidelobe apodization," IEEE Transactions on Image Processing, vol. 14, no. 4, pp. 537-547, April 2005.

[7] P. López-Dekker and J. J. Mallorquí, "Capon-and APES-based SAR processing: Performance and practical considerations," IEEE Transactions on Geoscience and Remote Sensing, vol. 48, no. 5, pp. 2388-2402, 2010.

[8] J. Capon, "High-resolution frequency-wavenumber spectrum analysis," Proceedings of the IEEE, vol. 57, no. 8, pp. 1408-1418, 1969. 
[9] S. R. DeGraaf, "SAR imaging via modern 2-D spectral estimation methods," IEEE Transactions on Image Processing, vol. 7, no. 5, pp. 729-761, 1998.

[10] T. Bianchi, F. Argenti, and L. Alparone, "Segmentation-based MAP despeckling of SAR images in the undecimated wavelet domain," IEEE Transactions on Geoscience and Remote Sensing, vol. 46, no. 9, pp. 2728-2742, 2008

[11] R. K. Raney, "Synthetic aperture imaging radar and moving targets," IEEE Transactions on Aerospace and Electronic Systems, no. 3, pp. 499-505, 1971.

[12] J. R. Fienup, "Detecting moving targets in SAR imagery by focusing," IEEE Transactions on Aerospace and Electronic Systems, vol. 37, no. 3 , pp. 794-809, 2001.

[13] M. Kirscht, "Detection and imaging of arbitrarily moving targets with single-channel SAR," IEE Proceedings - Radar, Sonar and Navigation, vol. 150 , no. 1 , pp. $7-11,2003$

[14] S. Zhu, G. Liao, Y. Qu, Z. Zhou, and X. Liu, "Ground moving targets imaging algorithm for synthetic aperture radar," IEEE Transactions on Geoscience and Remote Sensing, vol. 49, no. 1, pp. 462-477, 2011.

[15] A. Budillon, A. Evangelista, and G. Schirinzi, "GLRT detection of moving targets via multibaseline along-track interferometric SAR systems," IEEE Geoscience and Remote Sensing Letters, vol. 9, no. 3, pp. 348352, May 2012.

[16] P. Runkle, L. H. Nguyen, J. H. McClellan, and L. Carin, "Multi-aspect target detection for SAR imagery using hidden Markov models," IEEE Transactions on Geoscience and Remote Sensing, vol. 39, no. 1, pp. 46-55, Jan 2001.

[17] L. M. Novak, M. C. Burl, W. Irving, and G. Owirka, "Optimal polarimetric processing for enhanced target detection," in Proceedings of Telesystems Conference, vol. 1. IEEE, 1991, pp. 69-75.

[18] J.-C. Souyris, C. Henry, and F. Adragna, "On the use of complex SAR image spectral analysis for target detection: Assessment of polarimetry," IEEE Transactions on Geoscience and Remote Sensing, vol. 41, no. 12, pp. 2725-2734, 2003.

[19] H. Li, W. Perrie, Y. He, S. Lehner, and S. Brusch, "Target detection on the ocean with the relative phase of compact polarimetry SAR," IEEE Transactions on Geoscience and Remote Sensing, vol. 51, no. 6, pp. 3299-3305, 2013.

[20] A. Ferretti, C. Prati, and F. Rocca, "Permanent scatterers in SAR interferometry," IEEE Transactions on Geoscience and Remote Sensing, vol. 39, no. 1, pp. 8-20, 2001.

[21] A. Ferretti, A. Fumagalli, F. Novali, C. Prati, F. Rocca, and A. Rucci, "A new algorithm for processing interferometric data-stacks: SqueeSAR," IEEE Transactions on Geoscience and Remote Sensing, vol. 49, no. 9, pp. 3460-3470, 2011

[22] J. Högbom, "Aperture synthesis with a non-regular distribution of interferometer baselines," Astronomy and Astrophysics Supplement Series, vol. 15 , p. 417,1974

[23] J. Tsao and B. Steinberg, "Reduction of sidelobe and speckle artifacts in microwave imaging: the CLEAN technique," IEEE Transactions on Antennas and Propagation, vol. 36, no. 4, 1988.

[24] K. Kulpa, "The CLEAN type algorithms for radar signal processing," in 2008 Microwaves, Radar and Remote Sensing Symposium, Sept 2008, pp. 152-157.

[25] J. Li and P. Stoica, "Efficient mixed-spectrum estimation with applications to target feature extraction," IEEE Transactions on Signal Processing, vol. 44, no. 2, pp. 281-295, 1996.

[26] L. Denis, F. Tupin, and X. Rondeau, "Exact discrete minimization for TV+L0 image decomposition models," in Proceedings of the International Conference on Image Processing (ICIP). IEEE, 2010, pp. 25252528.

[27] S. Lobry, L. Denis, and F. Tupin, "Multitemporal SAR image decomposition into strong scatterers, background, and speckle," IEEE Journal of Selected Topics in Applied Earth Observations and Remote Sensing, vol. 9, no. 8, pp. 3419-3429, 2016

[28] R. Abergel, S. Ladjal, F. Tupin, and J.-M. Nicolas, "A complex spectrum based SAR image resampling method with restricted target sidelobes and statistics preservation," in IEEE International Geoscience and Remote Sensing Symposium (IGARSS), 2017.

[29] M. Eineder, T. Fritz, J. Mittermayer, A. Roth, E. Boerner, and H. Breit, "TerraSAR-X Ground Segment, Basic Product Specification Document," Defense Technical Information Center, Tech. Rep., 2008, issue 1.5.

[30] C. E. Shannon, "Communication in the presence of noise," Proceedings of the Institute of Radio Engineers, vol. 37, no. 1, pp. 10-21, 1949.

[31] E. T. Whittaker, "On the functions which are represented by the expansion of interpolating theory," in Proceedings of the Royal Society of Edinburgh, vol. 35, 1915, pp. 181-194.
[32] R. Abergel and L. Moisan, "The Shannon Total Variation," Journal of Mathematical Imaging and Vision, vol. 59, no. 2, pp. 341-370, 2017.

[33] J. W. Goodman, "Statistical properties of laser speckle patterns," Laser speckle and related phenomena, vol. 9, pp. 9-75, 1975.

[34] C. Oliver and S. Quegan, Understanding Synthetic Aperture Radar Images. SciTech Publishing, 2004.

[35] U. J. Schwarz, "Mathematical-statistical description of the iterative beam removing technique (method CLEAN)," Astronomy and Astrophysics, vol. 65, p. $345,1978$.

[36] B. Clark, "An efficient implementation of the algorithm 'CLEAN'," Astronomy and Astrophysics, vol. 89, p. 377, 1980.

[37] S. G. Mallat and Z. Zhang, "Matching pursuits with time-frequency dictionaries," IEEE Transactions on Signal Processing, vol. 41, no. 12, pp. 3397-3415, 1993

[38] Y. C. Pati, R. Rezaiifar, and P. S. Krishnaprasad, "Orthogonal matching pursuit: Recursive function approximation with applications to wavelet decomposition," in Conference Record of The Twenty-Seventh Asilomar Conference on Signals, Systems and Computers. IEEE, 1993, pp. 4044

[39] G. Gao, L. Liu, L. Zhao, G. Shi, and G. Kuang, "An adaptive and fast CFAR algorithm based on automatic censoring for target detection in high-resolution," IEEE transactions on Geoscience and Remote Sensing, vol. 47, no. 6, pp. 1685-1697, 2009.

[40] S. Kuttikkad and R. Chellappa, "Non-gaussian CFAR techniques for target detection in high resolution SAR images," in Proceedings of the International Conference on Image Processing (ICIP), vol. 1. IEEE, 1994, pp. 910-914.

[41] A. Lopes, E. Nezry, R. Touzi, and H. Laur, "Structure detection and statistical adaptive speckle filtering in SAR images," International Journal of Remote Sensing, vol. 14, no. 9, pp. 1735-1758, 1993.

[42] L. Novak and S. Hesse, "On the performance of order-statistics CFAR detectors," in Conference Record of the Twenty-Fifth Asilomar Conference on Signals, Systems and Computers. IEEE, 1991, pp. 835-840.

[43] A. Desolneux, L. Moisan, and J.-M. Morel, From gestalt theory to image analysis: a probabilistic approach. Springer Science \& Business Media, 2007, vol. 34.

[44] A. Desolneux, L. Moisan, and J.-M. Morel, "Meaningful alignments," International Journal of Computer Vision, vol. 40, no. 1, pp. 7-23, 2000.

[45] J. Delon, A. Desolneux, J.-L. Lisani, and A. B. Petro, "A nonparametric approach for histogram segmentation," IEEE Transactions on Image Processing, vol. 16, no. 1, pp. 253-261, 2007.

[46] P. Musé, F. Sur, F. Cao, Y. Gousseau, and J.-M. Morel, "An a contrario decision method for shape element recognition," International Journal of Computer Vision, vol. 69, no. 3, pp. 295-315, 2006.

[47] J. Lezama, R. Grompone von Gioi, G. Randall, and J.-M. Morel, "Finding vanishing points via point alignments in image primal and dual domains," in Proceedings of the IEEE Conference on Computer Vision and Pattern Recognition, 2014, pp. 509-515.

[48] A. Desolneux, L. Moisan, and J.-M. Morel, "Edge detection by Helmholtz principle," Journal of Mathematical Imaging and Vision, vol. 14 , no. 3, pp. 271-284, 2001.

[49] R. Grompone von Gioi, J. Jakubowicz, J.-M. Morel, and G. Randall, "LSD: A fast line segment detector with a false detection control," IEEE Transactions on Pattern Analysis and Machine Intelligence, vol. 32, no. 4 , pp. 722-732, 2010.

[50] A. Robin, L. Moisan, and S. Le Hegarat-Mascle, "An a-contrario approach for subpixel change detection in satellite imagery," IEEE Transactions on Pattern Analysis and Machine Intelligence, vol. 32, no. 11, pp. 1977-1993, 2010.

[51] F. Dellinger, J. Delon, Y. Gousseau, J. Michel, and F. Tupin, "SARSIFT: a SIFT-like algorithm for SAR images," IEEE Transactions on Geoscience and Remote Sensing, vol. 53, no. 1, pp. 453-466, 2015.

[52] D. Lowe, Perceptual organization and visual recognition. Springer Science \& Business Media, 2012, vol. 5.

[53] B. Grosjean and L. Moisan, "A-contrario detectability of spots in textured backgrounds," Journal of Mathematical Imaging and Vision, vol. 33, no. 3, pp. 313-337, 2009. 